Physica Scripta, Vol. 77, № 2, art. 025004 (2008)

\title{
Higher-order approximate solutions to the relativistic and Duffing-harmonic oscillators by modified He's homotopy methods
}

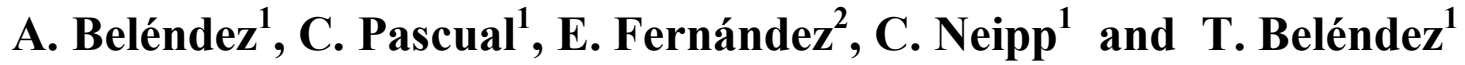 \\ ${ }^{1}$ Departamento de Física, Ingeniería de Sistemas y Teoría de la Señal. \\ Universidad de Alicante. Apartado 99. E-03080 Alicante. SPAIN \\ ${ }^{2}$ Departamento de Óptica, Farmacología y Anatomía. \\ Universidad de Alicante. Apartado 99. E-03080 Alicante. SPAIN \\ E-mail: a.belendez@ua.es
}

Corresponding author: A. Beléndez

Phone: +34-96-5903651

Fax: +34-96-5909750 


\section{ABSTRACT}

A modified He's homotopy perturbation method is used to calculate higher-order analytical approximate solutions to the relativistic and Duffing-harmonic oscillators. The He's homotopy perturbation method is modified by truncating the infinite series corresponding to the first order approximate solution before introducing this solution in the second order linear differential equation, and so on. We find this modified homotopy perturbation method works very well for the whole range of initial amplitudes, and the excellent agreement of the approximate frequencies and periodic solutions with the exact ones has been demonstrated and discussed. The approximate formulas obtained show excellent agreement with the exact solutions, and are valid for small as well as large amplitudes of oscillation, including the limiting cases of amplitude approaching zero and infinity. For the relativistic oscillator, only one iteration leads to high accuracy of the solutions with a maximal relative error for the approximate frequency of less than $1.6 \%$ for small and large values of oscillation amplitude, while this relative error is $0.65 \%$ for two iterations with two harmonics and as low as $0.18 \%$ when three harmonics are considered in the second approximation. For the Duffing-harmonic oscillator the relative error is as low as $0.078 \%$ when the second approximation is considered. Comparison of the result obtained using this method with those obtained by the harmonic balance methods reveals that the former is very effective and convenient.

Keywords: Nonlinear oscillator; Relativistic oscillator; Duffing-harmonic oscillator; Approximate solutions; Homotopy perturbation method.

PACS numbers: 02.30.Jr, 02.30.Lt, 02.30.-f 


\section{Introduction}

Considerable attention has been directed towards the study of strongly nonlinear oscillators and several methods have been used to find approximate solutions to nonlinear problems, such as perturbation techniques [1-6], variational methods [7-13] or harmonic balance based methods [14-21]. An excellent review of some asymptotic methods for strongly nonlinear equations can be found in detail in references [22] and [23]. In general, given the nature of a nonlinear phenomenon, the approximate methods can only be applied within certain ranges of the physical parameters and to certain classes of problems.

The purpose of this paper is to calculate higher-order analytical approximations to the periodic solutions to the relativistic and Duffing-harmonic oscillators. The relativistic oscillator is a nonlinear oscillator whose nonlinearity (anharmonicity) is a relativistic effect. When the energy of a simple harmonic oscillator is such that the velocities become relativistic, the simple harmonic motion (linear oscillations) at low energy becomes anharmonic (nonlinear oscillations) at high energy. Then, the strength of the nonlinearity increases with increasing total relativistic energy, and in the non-relativistic limit the oscillator becomes linear. Duffing-harmonic oscillator. This oscillator is a conservative non-linear oscillatory system modelled by a potential having a rational form for the potential energy [41]. This system leads to a differential equation for which the usual, expansion in a small parameter, perturbation procedures do not apply because a linear term and a perturbation parameter are not present.

The homotopy perturbation method [22-38], which requires neither a small parameter nor a linear term in a differential equation, yields a very rapid convergence of the solution series; in most cases only one iteration leads to high accuracy of the solution. This method provides an effective and convenient mathematical tool for nonlinear differential equations [22]. To obtain higher-order analytical approximate periodic solutions for the relativistic and Duffing-harmonic oscillators, we apply a modified He's homotopy perturbation method (HHPM). Applying the standard He's homotopy perturbation method (HHPH) to this oscillator, an infinite series is obtained for the first analytical approximate solution $[39,40]$ and this series must be introduced in the linear differential equation to obtain the second-order approximate solution. However, since it is 
difficult to work with an infinite series, we truncate this series before solving the subsequent linear differential equation considering only two harmonics for the second order approximation, three harmonics for the third order approximation, and so on. In this sense, the truncated approximate solutions have the same form as those considered when harmonic balance methods are applied. As we can see, the results presented in this paper reveal that the method is very effective and convenient for conservative nonlinear oscillators for which the restoring force has a non-polynomial form.

He's homotopy perturbation method changes a difficult problem into a simple problem which can be easily solved. This method, in contrast to the traditional perturbation methods, requires neither a small parameter nor a linear term in a differential equation. In He's technique a homotopy with an imbedding parameter $p \in[0,1]$ is constructed, and this parameter is considered 'small parameter', so the method is called the homotopy perturbation method, which can take full advantage of the traditional perturbation and homotopy technique [22, 38]. This perturbation approach has been applied not only to nonlinear oscillators but also to other nonlinear problems. This technique yields a very rapid convergence of the solution series; in most cases only one iteration leads to high accuracy of the solution. As we will see, some of the most interesting features of He's homotopy perturbation method are its simplicity and its very good accuracy in whole range of amplitude of oscillation.

\section{Relativistic oscillator}

\section{1.- Solution procedure}

The governing non-dimensional equation of motion for the relativistic oscillator is [41]

$$
x^{\prime \prime}+\left(1-x^{2}\right)^{3 / 2} x=0
$$

where $x$ and $t$ are dimensionless variables and $x^{\prime}=\mathrm{d} x / \mathrm{d} t$ and $x^{\prime \prime}=\mathrm{d}^{2} x / \mathrm{d} t^{2}$.

Eq. (1) is based on taking the rest mass of the particle, m, and the elastic constant, $k$, of the one-dimensional harmonic oscillator force $F(\tilde{x})=-k \tilde{x}$ (Hooke's force law), to be unity, where $\tilde{x}$ is a dimensional variable. Eq. (1) is obtainable from Newton's equation of 
motion in the form $F=\mathrm{d} p / \mathrm{d} \tilde{t}$, by replacing the Newtonian momentum $p$ with its relativistic form $p=m v\left(1-v^{2} / c^{2}\right)^{-1 / 2}$, where $v=\mathrm{d} \tilde{x} / \mathrm{d} \tilde{t}$ is the speed of the particle, $c$ is the speed of light and $\tilde{t}$ is the coordinate time (dimensional variable) related with the proper time $\tau$ by $\mathrm{d} \tilde{t}=\left(1-v^{2} / c^{2}\right)^{-1 / 2} \mathrm{~d} \tau$. We can easily verify that the dimensionless length $x$ and the dimensionless time $t$ are related to the dimensional variables $\tilde{x}$ and $\tilde{t}$ through $x=\omega_{0} \tilde{x} / c$ and $t=\omega_{0} \tilde{t}$, respectively, where $\omega_{0}=\sqrt{k / m}$ is the angular frequency for the non-relativistic oscillator (linear oscillator).

On the other hand, the even power term in Eq. (1), $x^{2}=(\mathrm{d} x / \mathrm{d} t)^{2}$ acts like the powers of coordinates in that it does not cause a damping of the amplitude of oscillations with time. Therefore, Eq. (1) is an example of a generalized conservative system [14]. At the limit when $(\mathrm{d} x / \mathrm{d} t)^{2}<<1$, Eq. (1) becomes $x^{\prime \prime}+x=0$, the oscillator is linear and the proper time $\tau$ becomes equivalent to the coordinate time $t$ to this order.

Introducing the phase space variable $(x, y)$, Eq. (1) can be written in the system form

$$
x^{\prime}=y, \quad y^{\prime}=-\left(1-y^{2}\right)^{3 / 2} x
$$

Consequently, the trajectories in phase space are given by solutions to the first order, ordinary differential equation

$$
\frac{\mathrm{d} y}{\mathrm{~d} x}=-\frac{\left(1-y^{2}\right)^{3 / 2} x}{y}
$$

As Mickens pointed out, since the physical solution of both Eq. (1) and Eq. (3) are real, the phase space has a "strip" structure [41], i.e.,

$$
-\infty<x<+\infty, \quad-1<y<+1
$$

Then unlike the usual non-relativistic harmonic oscillator, the relativistic oscillator is bounded in the $y$ variable. Mickens has proved that all the trajectories to Eq. (3) are 
closed in the open region of phase space given by Eq. (4) and then all the physical solutions to Eq. (1) are periodic [41].

The homotopy perturbation method can now be applied to obtain analytic approximations to the periodic solutions of Eq. (1). First we make a change of variable, $y \rightarrow u$, such that $-\infty<u<+\infty$. The required transformation is [41]

$$
y=\frac{u}{\sqrt{1+u^{2}}}
$$

It follows that

$$
u^{\prime}=-x
$$

And then, the corresponding second order differential equation for $u$ is

$$
u^{\prime \prime}+\frac{u}{\sqrt{1+u^{2}}}=0
$$

which corresponds to a conservative nonlinear oscillator. We consider the following initial conditions to Eq. (7)

$$
u(0)=B, \quad u^{\prime}(0)=0
$$

Eq. (7) can be re-written in the form

$$
u^{\prime \prime}+u=u-\frac{u}{\sqrt{1+u^{2}}}
$$

For Eq. (9) we can establish the following homotopy

$$
u^{\prime \prime}+1 \cdot u=p\left(u-\frac{u}{\sqrt{1+u^{2}}}\right)
$$


where $p$ is the homotopy parameter. When $p=0$, Eq. (10) becomes a linearized equation and for the case $p=1$, Eq. (10) becomes the original problem. Now the homotopy parameter $p$ is used to expand the solution $u(t)$ in powers of the parameter $p$

$$
\begin{gathered}
u(t)=u_{0}(t)+p u_{1}(t)+p^{2} u_{2}(t)+\ldots \\
1=\omega^{2}-p \alpha_{1}-p^{2} \alpha_{2}-p^{3} \alpha_{3}-\ldots
\end{gathered}
$$

where $\alpha_{i}(i=1,2, \ldots)$ are to be determined and where $\omega$ is the unknown angular frequency of the nonlinear oscillator. Substituting Eqs. (11) and (12) into Eq. (10), and equating the terms with identical powers of $p$, we can obtain a series of linear equations, of which we write only the first three

$u_{0}^{\prime \prime}+\omega^{2} u_{0}=0, \quad u_{0}(0)=B, u_{0}^{\prime}(0)=0$

$u_{1}^{\prime \prime}+\omega^{2} u_{1}=\left(1+\alpha_{1}\right) u_{0}-\frac{u_{0}}{\sqrt{1+u_{0}^{2}}}, \quad u_{1}(0)=0, \quad u_{1}^{\prime}(0)=0$

$u_{2}^{\prime \prime}+\omega^{2} u_{2}=\alpha_{2} u_{0}+\left(1+\alpha_{1}\right) u_{1}-\frac{u_{1}}{\left(1+u_{0}^{2}\right)^{3 / 2}}, \quad u_{2}(0)=0, \quad u_{2}^{\prime}(0)=0$

In these equations we have taken into account the following expression

$$
f(u)=f\left(u_{0}+p u_{1}+p^{2} u_{2}+\ldots\right)=f\left(u_{0}\right)+p u_{1} f^{\prime}\left(u_{0}\right)+p^{2}\left\lfloor u_{2} f^{\prime}\left(u_{0}\right)+\frac{1}{2} u_{1}^{2} f^{\prime \prime}\left(u_{0}\right)\right\rfloor+O\left(p^{3}\right)
$$

where 


$$
f(u)=\frac{u}{\sqrt{1+u^{2}}}
$$

The solution of Eq. (13) is

$$
u_{0}(t)=B \cos \omega t
$$

Substituting Eq. (18) into Eq. (14), we obtain the following differential equation for $u_{1}$

$$
\frac{\mathrm{d}^{2} u_{1}}{\mathrm{~d} t^{2}}+\omega^{2} u_{1}=\left(1+\alpha_{1}\right) B \cos \omega t-\frac{B \cos \omega t}{\sqrt{1+B^{2} \cos ^{2} \omega t}}
$$

It is possible to do the following Fourier series expansion

$$
\frac{B \cos \omega t}{\sqrt{1+B^{2} \cos ^{2} \omega t}}=\sum_{n=0}^{\infty} a_{2 n+1} \cos [(2 n+1) \omega t]
$$

where the first term of this expansion can be obtained by means of the following equation

$$
a_{1}=\frac{4}{\pi} \int_{0}^{\pi / 2} \frac{B \cos \theta}{\sqrt{1+B^{2} \cos ^{2} \theta}} \cos \theta \mathrm{d} \theta=\frac{4}{\pi B}\left[E\left(-B^{2}\right)-K\left(-B^{2}\right)\right]
$$

where $K(m)$ and $E(m)$ are the complete elliptic integrals of the first and second kind, respectively, defined as follows [42]

$$
\begin{gathered}
K(m)=\int_{0}^{\pi / 2} \frac{\mathrm{d} \theta}{\sqrt{1-m \sin ^{2} \theta}} \\
E(m)=\int_{0}^{\pi / 2} \sqrt{1-m \sin ^{2} \theta} \mathrm{d} \theta
\end{gathered}
$$


Substitution of Eq. (20) into Eq. (19) gives

$$
\frac{\mathrm{d}^{2} u_{1}}{\mathrm{~d} t^{2}}+\omega^{2} u_{1}=\left(1+\alpha_{1}-\frac{a_{1}}{B}\right) B \cos \omega t-\sum_{n=1}^{\infty} a_{2 n+1} \cos [(2 n+1) \omega t]
$$

If there are to be no secular terms in $u_{1}(t)$, contributions proportional to $\cos \omega t$ on the right of Eq. (24) must be eliminated

$$
1+\alpha_{1}-\frac{a_{1}}{B}=0
$$

Substituting Eq. (21) into Eq. (25) and reordering, we can easily find that the solution $\alpha_{1}$ is

$$
\alpha_{1}=-1+\frac{4}{\pi B^{2}}\left[E\left(-B^{2}\right)-K\left(-B^{2}\right)\right]
$$

From Eqs. (12) and (26), writing $p=1$, we can easily find that the first order approximate frequency is

$$
\omega_{1}(B)=\frac{2}{\sqrt{\pi} B} \sqrt{E\left(-B^{2}\right)-K\left(-B^{2}\right)}
$$

We re-write Eq. (24) in the form

$$
\frac{\mathrm{d}^{2} u_{1}}{\mathrm{~d} t^{2}}+\omega^{2} u_{1}=-\sum_{n=1}^{\infty} a_{2 n+1} \cos [(2 n+1) \omega t]
$$

where the coefficients $a_{2 n+1}$ are obtained by means of the following equation 


$$
a_{2 n+1}=\frac{4}{\pi} \int_{0}^{\pi / 2} \frac{B \cos \theta}{\sqrt{1+B^{2} \cos ^{2} \theta}} \cos [(2 n+1) \theta] \mathrm{d} \theta
$$

The solution of Eq. (28) can be written as follows

$$
u_{1}(t)=\sum_{n=0}^{\infty} d_{2 n+1} \cos [(2 n+1) \omega t]
$$

Substituting Eq. (30) into Eq. (28) gives

$$
-\sum_{n=1}^{\infty} \omega^{2} 4 n(n+1) d_{2 n+1} \cos [(2 n+1) \omega t]=-\sum_{n=1}^{\infty} a_{2 n+1} \cos [(2 n+1) \omega t]
$$

and we can easily obtain

$$
d_{2 n+1}=\frac{a_{2 n+1}}{4 n(n+1) \omega^{2}}
$$

for $n \geq 1$. Taking into account that $u_{1}(0)=0$, Eq. (30) gives

$$
u_{1}(0)=\sum_{n=0}^{\infty} d_{2 n+1}=0
$$

and the value of coefficient $d_{1}$ is given by the following expression

$$
d_{1}=-\sum_{n=1}^{\infty} d_{2 n+1}=-\frac{1}{4 \omega^{2}} \sum_{n=1}^{\infty} \frac{a_{2 n+1}}{n(n+1)}
$$


To determine the second-order approximate solution it is necessary to substitute Eq. (30) into Eq. (15). Then secular terms are eliminated and parameter $\alpha_{2}$ can be calculated. However, it is difficult to solve the new differential equation because, as $u_{1}(t)$ has an infinite number of harmonics, it would be necessary to multiply this infinite series by $-\left(1+u_{0}^{2}\right)^{-3 / 2}$. At this moment we introduce a modification in He's homotopy perturbation method to simplify the solution procedure [36]. $u_{1}(t)$ has an infinite number of harmonics, however we can truncate the series expansion at Eq. (30) and write an approximate equation $u_{1}^{(N)}(t)$ in the form

$$
u_{1}^{(N)}=\sum_{n=0}^{N} d_{2 n+1} \cos [(2 n+1) \omega t]
$$

which has only a finite number of harmonics. Comparing Eqs. (30) and (35), it follows that

$$
\lim _{N \rightarrow \infty} u_{1}^{(N)}(t)=u_{1}(t)
$$

In the simplest case we consider $N=1(n=0,1)$ and Eq. (35) becomes

$$
u_{1}^{(1)}(t)=-d_{3}(\cos \omega t-\cos 3 \omega t)=4 d_{3}\left(\cos \omega t-\cos ^{3} \omega t\right)
$$

which has a similar form than the second order approximate solution considered in harmonic balance methods [34]. From Eqs. (29) and (32) the following expression for the coefficient $d_{3}$ is obtained

$$
d_{3}=-d_{1}=\frac{a_{3}}{8 \omega^{2}}=\frac{\left(8+B^{2}\right) E\left(-B^{2}\right)-\left(8+5 B^{2}\right) K\left(-B^{2}\right)}{6 \pi B^{3} \omega^{2}}
$$

where 
$a_{3}=\frac{4}{\pi} \int_{0}^{\pi / 2} \frac{B \cos \theta}{\sqrt{1+B^{2} \cos ^{2} \theta}} \cos 3 \theta \mathrm{d} \theta=\frac{4\left[\left(8+B^{2}\right) E\left(-B^{2}\right)-\left(8+5 B^{2}\right) K\left(-B^{2}\right)\right]}{3 \pi B^{3}}$

Substitution of Eq. (37) into Eq. (15) gives the following equation for gives the following equation for $u_{2}(t)$

$$
u_{2}^{\prime \prime}+\omega^{2} u_{2}=\alpha_{2} u_{0}+\left(1+\alpha_{1}\right) u_{1}^{(1)}-\frac{u_{1}^{(1)}}{\left(1+u_{0}^{2}\right)^{3 / 2}}
$$

and taking into account Eqs. (32) and (37), Eq. (40) becomes

$$
u_{2}^{\prime \prime}+\omega^{2} u_{2}=\alpha_{2} B \cos \omega t-\frac{a_{3}\left(1+\alpha_{1}\right)}{2 \omega^{2}}\left(\cos \omega t-\cos ^{3} \omega t\right)+\frac{a_{3}\left(\cos \omega t-\cos ^{3} \omega t\right)}{2 \omega^{2}\left(1+B^{2} \cos ^{2} \omega t\right)^{3 / 2}}
$$

The secular term in the solution for $u_{2}(t)$ can be eliminated if

$$
\int_{0}^{\pi / 2}\left\lfloor\alpha_{2} B \cos \theta-\frac{a_{3}\left(1+\alpha_{1}\right)}{2 \omega^{2}}\left(\cos \theta-\cos ^{3} \theta\right)+\frac{a_{3}\left(\cos \theta-\cos ^{3} \theta\right)}{2 \omega^{2}\left(1+B^{2} \cos ^{2} \theta\right)^{3 / 2}}\right\rfloor \cos \theta \mathrm{d} \theta=0
$$

The integration of Eq. (42) gives

$$
\frac{\pi a_{3}}{32 \omega^{2}}\left(1+\alpha_{1}\right)-\frac{\pi}{4} B \alpha_{2}+\frac{a_{3}}{\omega^{2} B^{4}} E(-B)-\frac{\left(2+B^{2}\right) a_{3}}{2 \omega^{2} B^{4}} K\left(-B^{2}\right)=0
$$

Substituting Eqs. (26), (38) and (39) into Eq. (36), and reordering, Eq. (36) can be solved for $\alpha_{2}$, that is

$$
\alpha_{2}=-\frac{2\left[\left(8+B^{2}\right) E\left(-B^{2}\right)-\left(8+5 B^{2}\right) K\left(-B^{2}\right)\right]^{2}}{3 \pi^{2} B^{8} \omega^{2}}
$$


From Eqs. (12), (26) and (44), and taking $p=1$, one can easily obtain the following expression for the second order approximate frequency is

$$
\omega_{2}^{(1)}(B)=\sqrt{\frac{6 B^{2}\left[E\left(-B^{2}\right)-K\left(-B^{2}\right)\right]+\sqrt{6 \Delta}}{3 \pi B^{4}}}
$$

where

$$
\begin{aligned}
\Delta(B) & =\left(-64-16 B^{2}+5 B^{4}\right) E^{2}\left(-B^{2}\right) \\
& +\left(128+96 B^{2}-10 B^{4}\right) E\left(-B^{2}\right) K\left(-B^{2}\right)-\left(64+80 B^{2}+19 B^{4}\right) K^{2}\left(-B^{2}\right)
\end{aligned}
$$

In references [39] and [40] it was proved that the simplest approximation for $u(t)$ is

$$
u(t)=u_{0}(t)+u_{1}(t) \approx u_{0}(t)=B \cos \omega_{j} t, \quad j=1,2
$$

gives very good results for $x(t)$. In order to find an approximate expression for $x_{j}(t)$ we use Eqs. (5) and (47) and obtain

$$
y_{j}(t) \approx \frac{B \cos \omega_{j} t}{\sqrt{1+B^{2} \cos ^{2} \omega_{j} t}}, \quad j=1,2
$$

However, we should not forget that we are really looking for is an approximate analytical solution to Eq. (1), that is, $x(t)$. In order to find an approximate expression for $x_{j}(t) \quad(j=$ 1,2) we integrate Eq. (48) taking into account that $y=\mathrm{d} x / \mathrm{d} t$ and we obtain

$$
x_{j}(t)=\frac{1}{\omega_{j}(B)} \sin ^{-1}\left[\frac{B}{\sqrt{1+B^{2}}} \sin \left[\omega_{j}(B) t\right]\right]
$$

Moreover, it is convenient to express the approximate angular frequency and the solution in terms of oscillation amplitude $A$ rather than as a function of $B$. It is now necessary to 
find a relation between oscillation amplitude $A$ and parameter $B$ used to solve Eq. (1) approximately. From Eq. (3) we get

$$
\frac{1}{\left(1-y^{2}\right)^{1 / 2}}+\frac{1}{2} x^{2}=C
$$

where $C$ is a constant to be determined as a function of initial conditions. From Eqs. (5) and (8) we can easily obtain $C=\left(1+B^{2}\right)^{1 / 2}$ and Eq. (50) can be written as follows

$$
\frac{1}{\left(1-y^{2}\right)^{1 / 2}}+\frac{1}{2} x^{2}=\left(1+B^{2}\right)^{1 / 2}
$$

In addition, when $x=A$, the velocity $y=\mathrm{d} x / \mathrm{d} t$ is zero. Taking this into account in Eq. (51), we obtain the following relation between amplitude $A$ and parameter $B$

$$
1+\frac{1}{2} A^{2}=\left(1+B^{2}\right)^{1 / 2}
$$

From the above equation we can easily find that the solution for $B$ is

$$
B=A\left(1+\frac{1}{4} A^{2}\right)^{1 / 2}
$$

Substituting Eq. (53) into Eqs. (27) and (45) and we can obtain the first- and second-order approximate angular frequencies, $\omega_{1}(A)$ and $\omega_{2}(A) \equiv \omega_{2}^{(1)}(A)$, respectively, for the relativistic oscillator as a function of the oscillation amplitude $A$

$$
\omega_{1}(A)=\frac{4}{A} \sqrt{\frac{E(\zeta)-K(\zeta)}{\pi\left(4+A^{2}\right)}}
$$


$\omega_{2}(A)=\frac{2}{\sqrt{3 \pi} A^{4}\left(4+A^{2}\right)^{2}}\left[\left(384 A^{6}+288 A^{8}+72 A^{10}+6 A^{12}\right)(E(\zeta)-K(\zeta))+\sqrt{6} A^{4}\left(4+A^{2}\right)^{2} \sqrt{\mathrm{Z}}\right]^{1 / 2}$

where

$$
\zeta(A)=-\frac{1}{4} A^{2}\left(4+A^{2}\right)
$$

and

$$
\begin{aligned}
\mathrm{Z}(A) & =\left(-1024-256 A^{2}+16 A^{4}+40 A^{6}+5 A^{8}\right) E^{2}(\zeta) \\
& -2\left(-1024-768 A^{2}-176 A^{4}+8 A^{6}+A^{8}\right) E(\zeta) K(\zeta) \\
& -\left(1024+1280 A^{2}+624 A^{4}+152 A^{6}+19 A^{8}\right) K^{2}(\zeta)
\end{aligned}
$$

The approximate solution $x_{j}(t)$ is obtained by substituting Eqs. (54) or (55) and Eq. (53) in Eq. (49)

$$
x_{j}(t)=\frac{1}{\omega_{j}(A)} \sin ^{-1}\left[\sqrt{\frac{4 A^{2}+A^{4}}{4+4 A^{2}+A^{4}}} \sin \left[\omega_{j}(A) t\right]\right]
$$

where $\omega_{j}(A)(j=1,2)$ are obtained from Eqs. (54) and (55).

Since the dimensionless variable $y$ is equal to $v / c$, where $v$ is the particle velocity and $c$ the velocity of light, from Eqs. (51) and (53) we have

$$
\beta_{0}=y(0)=\frac{v_{0}}{c}=\frac{B}{\sqrt{1+B^{2}}}=\sqrt{\frac{4 A^{2}+A^{4}}{4+4 A^{2}+A^{4}}}
$$

\section{2.- Results and discussion}

In this section we illustrate the accuracy of the proposed approach by comparing the second-order approximate frequency $\omega_{2}(A)$ (Eq. (55)) obtained using the modify 
homotopy perturbation method considered in this paper, with the exact frequency $\omega_{e}(A)$ and with the first-order approximate frequency $\omega_{1}(A)$ (Eq. (54)). The exact angular frequency is calculated as follows. Substituting Eq. (53) into Eq. (51), we obtain

$$
\frac{1}{\left(1-y^{2}\right)^{1 / 2}}=\frac{1}{2}\left(A^{2}-x^{2}\right)
$$

The exact frequency can then be derived as follows

$$
\omega_{e}(A)=\frac{\pi}{2}\left[\int_{0}^{A} \frac{1+\frac{1}{2}\left(A^{2}-x^{2}\right)}{\sqrt{A^{2}-x^{2}+\frac{1}{4}\left(A^{2}-x^{2}\right)^{2}}} \mathrm{~d} x\right]^{-1}
$$

which can be written in terms of elliptical integrals as follows

$$
\omega_{e}(A)=2 \pi\left[4 \sqrt{4+A^{2}} E\left(\frac{A^{2}}{4+A^{2}}\right)-\frac{8}{\sqrt{4+A^{2}}} K\left(\frac{A^{2}}{4+A^{2}}\right)\right]^{-1}
$$

where $K(m)$ and $E(m)$ are the complete elliptic integrals of the first and second kind, respectively, defined in Eqs. (22) and (23), respectively.

For small values of the amplitude $A$ it is possible to take into account the following power series expansions

$$
\begin{aligned}
& \omega_{e}(A)=1-\frac{3}{16} A^{2}+\frac{51}{1024} A^{4}-\frac{233}{16384} A^{6}+\frac{17499}{4194304} A^{8}-\ldots \\
& \omega_{1}(A)=1-\frac{3}{16} A^{2}+\frac{54}{1024} A^{4}-\frac{278}{16384} A^{6}+\frac{24984}{4194304} A^{8}-\ldots \\
& \omega_{2}(A)=1-\frac{3}{16} A^{2}+\frac{51}{1024} A^{4}-\frac{239}{16384} A^{6}+\frac{19302}{4194304} A^{8}-\ldots
\end{aligned}
$$


which were carried out using MATHEMATICA. As can be seen, in the expansion of the angular frequency $\omega_{1}(A)$ (Eq. (64)), only the first two terms are the same as the first two terms of the equation obtained in the power-series expansion of the exact angular frequency $\omega_{e}(A)$ (Eq. (63)). Whereas, in the expansion of the angular frequency $\omega_{2}(A)$ (Eq. (65)), the first three terms are the same as the first three terms of the equation obtained in the power-series expansion of the exact frequency $\omega_{e}(A)$ (Eq. (63)). If we compare the fourth term in Eq. (65) with the fourth term in the series expansion of the exact frequency $\omega_{e}(A)$ (Eq. (63)), we can see that the relative error of the four term of series expansion of $\omega_{2}(A)$ is $2.6 \%$. This implies that the second order approximate angular frequency $\omega_{2}(A)$ obtained in this paper provides excellent approximations to the exact frequency $\omega_{e}(A)$.

For very large values of the amplitude $A$ it is possible to take into account the following power series expansions

$$
\begin{aligned}
& \omega_{e}(A)=\frac{1.57080}{A}+\ldots \\
& \omega_{1}(A)=\frac{1.59577}{A}+\ldots \\
& \omega_{2}(A)=\frac{1.56062}{A}+\ldots
\end{aligned}
$$

Once again we can see than the frequency $\omega_{2}(A)$ obtained in this paper provides excellent approximations to the exact frequency $\omega_{e}(A)$ for very large values of oscillation amplitude. Now, the relative errors of the first term of series expansions of $\omega_{1}(A)$ and $\omega_{2}(A)$ are $1.6 \%$ and $0.65 \%$, respectively. 
In Figure 1 we plotted the relative errors for the approximate frequencies $\omega_{1}(A)$ and $\omega_{2}(A)$. In this figure the percentage errors were calculated using the following equation

$$
\text { Relative error of } \omega_{j}(\%)=100\left|\frac{\omega_{j}-\omega_{e}}{\omega_{e}}\right|
$$

Figure 1 indicates that $\omega_{2}(A)$ provides excellent approximations to the exact frequency $\omega_{e}(A)$ for the range of values of oscillation amplitude.

Furthermore, we have the following equations

$$
\begin{gathered}
\lim _{A \rightarrow 0} \omega_{e}(A)=\lim _{A \rightarrow 0} \omega_{1}(A)=\lim _{A \rightarrow 0} \omega_{2}(A)=1 \\
\lim _{A \rightarrow \infty} \omega_{e}(A)=\lim _{A \rightarrow \infty} \omega_{1}(A)=\lim _{A \rightarrow \infty} \omega_{2}(A)=0 \\
\lim _{A \rightarrow \infty} \frac{\omega_{1}(A)}{\omega_{e}(A)}=1.01590 \\
\lim _{A \rightarrow \infty} \frac{\omega_{2}(A)}{\omega_{e}(A)}=0.99352
\end{gathered}
$$

Eqs. (70)-(73) illustrate very good agreement of the second order approximate frequency obtained in this paper, $\omega_{2}(A)$, with the exact frequency $\omega_{e}(A)$.

The exact periodic solutions $x(t)$ achieved by numerically integrating Eq. (1), and the proposed normalized second-order approximate periodic solution $x_{2}(t)$ in Eq. (58) for one complete cycle are plotted in Figures 2, 3, 4 and 5 for oscillation amplitudes $A=1,2$, 10 and 100, respectively. In these figures parameter $h$ is defined as follows 


$$
h=\frac{t}{T_{e}(A)}=\frac{\omega_{e}(A) t}{2 \pi}
$$

As we can see, in all cases, approximate solutions coincide with the exact one. Figures 2-5 show that Eq. (58) provides a good approximation to the exact periodic solutions and that the approximation $u(t) \approx u_{0}(t)=B \cos \omega_{j} t(j=1,2)$ considering in Eq. (47) is sufficient to calculate an approximate expression for $x(t)$. As we can see, for small values of $A$ (Figure 2) $x(t)$ is very close to the sine function form of non-relativistic simple harmonic motion. For $A \geq 2$ the curvature becomes more concentrated at the turning points $(x= \pm A)$. For these values of $A, x(t)$ becomes markedly anharmonic and is almost straight between the turning points. Only in the vicinity of the turning points, where the magnitude of the Hooke's law force is maximum and the velocity becomes relativistic, is the force effective in changing the velocity [43]. Figures 4 and 5 are two typical examples of the motion in the ultra-relativistic region where $\beta_{0} \rightarrow 1$.

\section{3.- A more accurate second-order approximate solution}

If we consider $N=2(n=0,1,2)$ in Eq. (35), we have

$$
u_{1}^{(2)}(t)=-d_{3}(\cos \omega t-\cos 3 \omega t)-d_{5}(\cos \omega t-\cos 5 \omega t)
$$

where

$$
\begin{gathered}
d_{5}=\frac{a_{5}}{24 \omega^{2}} \\
a_{5}=\frac{4}{15 \pi B^{5}}\left[\left(128+88 B^{2}+3 B^{4}\right) E\left(-B^{2}\right)-\left(128+152 B^{2}+39 B^{4}\right) K\left(-B^{2}\right)\right]
\end{gathered}
$$

and

$$
d_{1}=-\left(d_{3}+d_{5}\right)
$$


Substituting Eq. (75) into Eq. (15), eliminating the secular term in the solution for $u_{2}(t)$ and taking $p=1$ in Eq. (12), one can easily obtain the following expression for the second order approximate frequency

$$
\omega_{2}^{(2)}(B)=\sqrt{\frac{90 B^{4}\left[E\left(-B^{2}\right)-K\left(-B^{2}\right)\right]+2 \sqrt{15 F}}{45 \pi B^{6}}}
$$

where

$$
\begin{aligned}
F(B) & =\left(-8192-8192 B^{2}-3200 B^{4}-288 B^{6}+117 B^{8}\right) E^{2}\left(-B^{2}\right) \\
& +2\left(8192+12288 B^{2}+6784 B^{4}+1632 B^{6}-45 B^{8}\right) E\left(-B^{2}\right) K\left(-B^{2}\right) \\
& -\left(8192+16384 B^{2}+12416 B^{4}+4512 B^{6}+603 B^{8}\right) K^{2}\left(-B^{2}\right)
\end{aligned}
$$

Substituting Eq. (53) into Eqs. (79) and (80) we can obtain $\omega_{2}^{\prime} \equiv \omega_{2}^{(2)}$ as a function of $A$. For small values of the amplitude $A$ it is possible to take into account the following power series expansion

$$
\omega_{2}^{\prime}(A)=1-\frac{3}{16} A^{2}+\frac{51}{1024} A^{4}-\frac{233}{16384} A^{6}+\frac{17398}{4194304} A^{8}-\ldots
$$

As can be seen, in the expansion of the angular frequency $\omega_{2}^{\prime}(A)$ (Eq. (81)), the first four terms are the same as the first four terms of the equation obtained in the power-series expansion of the exact frequency $\omega_{e}(A)$ (Eq. (63)). If we compare the fifth term in Eq. (81) with the fifth term in the series expansion of the exact frequency $\omega_{e}(A)$ (Eq. (63)), we can see that the relative error of the fifth term of series expansion is less than $0.6 \%$. This implies that the second order approximate angular frequency $\omega_{2}^{\prime}(A)$ obtained in this paper provides excellent approximations to the exact frequency $\omega_{e}(A)$.

For very large values of the amplitude $A$ it is possible to take into account the following power series expansion 


$$
\omega_{2}^{\prime}(A)=\frac{1.56798}{A}+\ldots
$$

If we compare Eqs. (66) and (82), we can see than the frequency $\omega_{2}^{\prime}(A)$ obtained in this paper provides excellent approximations to the exact frequency $\omega_{e}(A)$ for very large values of oscillation amplitude. Now, the relative error of the first term of the series expansion of $\omega_{2}^{\prime}(A)$ is $0.18 \%$. Furthermore we have

$$
\lim _{A \rightarrow \infty} \frac{\omega_{2}^{\prime}(A)}{\omega_{e}(A)}=0.998205
$$

In Figure 6 we plotted the relative errors for the approximate frequencies $\omega_{2}(A)$ and $\omega_{2}^{\prime}(A)$. This figure indicates that $\omega_{2}^{\prime}(A)$ provides excellent approximations to the exact frequency $\omega_{e}(A)$ for the range of values of oscillation amplitude. The exact periodic solution $x(t)$ achieved by numerically integrating Eq. (1), and the proposed normalized second-order approximate periodic solution $x_{2}(t)$ for one complete cycle when $\omega_{2}^{\prime}(A)$ is considered is plotted in Figures 7 for $A=100$. As we can see the coincidence is complete. From these figures we can see that for large values of the amplitude, the normalized solutions appear to be composed of straight line segments.

\section{Duffing-harmonic oscillator}

\section{1.- Solution procedure}

The governing non-dimensional equation of motion for the Duffing-harmonic oscillator is [44]

$$
\frac{\mathrm{d}^{2} x}{\mathrm{~d} t^{2}}+\frac{x^{3}}{1+x^{2}}=0
$$

with the initial conditions

$$
x(0)=A \quad \text { and } \quad \frac{\mathrm{d} x}{\mathrm{~d} t}(0)=0
$$


Equation (84) is a conservative nonlinear oscillatory system having a rational form for the non-dimensional restoring force $F(x)$

$$
\frac{\mathrm{d}^{2} x}{\mathrm{~d} t^{2}}=F(x), \quad F(x)=-\frac{x^{3}}{1+x^{2}}
$$

It has been demonstrated that all the curves in the phase-space corresponding Eq. (84) are closed, an all motions for arbitrary initial conditions give periodic solutions [44]. We denote the angular frequency of these oscillations by $\omega$ and note that one of our major tasks is to determine $\omega(A)$, i.e., the functional behaviour of $\omega$ as a function of the initial amplitude.

For small $x$, the restoring force does have a dominant term proportional to $x$ and Eq. (84) approximates that of a truly Duffing-type nonlinear oscillator

$$
\frac{\mathrm{d}^{2} x}{\mathrm{~d} t^{2}}+x^{3}=0 \quad \text { for } x<<1
$$

and $\omega \approx 0.84721 A$ [45], which tends to zero when $A$ decreases. While for large $x$, the equation of motion approximates that of a linear harmonic oscillator

$$
\frac{\mathrm{d}^{2} x}{\mathrm{~d} t^{2}}+x=0 \quad \text { for } x>>1
$$

so, for large $A$, we have $\omega \approx 1$. Consequently the angular frequency $\omega$ increases from 0 to 1 as the initial value of $x(0)=A$ increases.

Equation (84) is not amenable to exact treatment and, therefore, approximate techniques must be resorted to. There is no small parameter in Eq. (84), so the standard perturbation methods cannot be applied directly. Due to the fact that the homotopy 
perturbation method requires neither a small parameter nor a linear term in a differential equation, one possibility to approximately solve Eq. (84) using the homotopy perturbation method.

Equation (84) can be re-written in the form

$$
\frac{\mathrm{d}^{2} x}{\mathrm{~d} t^{2}}+x=x-\frac{x^{3}}{1+x^{2}}
$$

For equation (89) we can establish the following homotopy

$$
\frac{\mathrm{d}^{2} x}{\mathrm{~d} t^{2}}+1 \cdot x=p\left(x-\frac{x^{3}}{1+x^{2}}\right)
$$

where $p$ is the homotopy parameter. When $p=0$, Eq. (90) becomes a linear differential equation for which an exact solution can be calculated; for $p=1$, Eq. (90) then becomes the original problem. Now the homotopy parameter $p$ is used to expand the solution $x(t)$ and the square of the unknown angular frequency $\omega$ as is shown in equations (11) and (12), where $\alpha_{i}(i=1,2, \ldots)$ are to be determined.

Substituting Eqs. (11) and (12) into Eq. (90) gives

$$
\begin{aligned}
& \left(x_{0}^{\prime \prime}+p x_{1}^{\prime \prime}+p^{2} x_{2}^{\prime \prime}+\ldots\right)+\left(\omega^{2}-p \alpha_{1}-p^{2} \alpha_{2}-\ldots\right)\left(x_{0}+p x_{1}+p^{2} x_{2}+\ldots\right) \\
& \quad=p\left[\left(x_{0}+p x_{1}+p^{2} x_{2}+\ldots\right)-\frac{\left(x_{0}+p x_{1}+p^{2} x_{2}+\ldots\right)^{3}}{1+\left(x_{0}+p x_{1}+p^{2} x_{2}+\ldots\right)^{2}}\right]
\end{aligned}
$$

and equating the terms with identical powers of $p$, we can obtain a series of linear equations, of which we write only the first three

$$
x_{0}^{\prime \prime}+\omega^{2} x_{0}=0, \quad x_{0}(0)=A, \quad x_{0}^{\prime}(0)=0
$$




$$
\begin{aligned}
& x_{1}^{\prime \prime}+\omega^{2} x_{1}=\left(1+\alpha_{1}\right) x_{0}-\frac{x_{0}^{3}}{1+x_{0}^{2}}, \quad x_{1}(0)=x_{1}^{\prime}(0)=0 \\
& x_{2}^{\prime \prime}+\omega^{2} x_{2}=\alpha_{2} x_{0}+\left(1+\alpha_{1}\right) x_{1}-\frac{\left(3+x_{0}^{2}\right) x_{0}^{2} x_{1}}{\left(1+x_{0}^{2}\right)^{2}}, \quad x_{2}(0)=x_{2}^{\prime}(0)=0
\end{aligned}
$$

In Eqs. (93) and (94) we have taken into account the following expression

$$
\begin{aligned}
f(x) & =f\left(x_{0}+p x_{1}+p^{2} x_{2}+\ldots\right)= \\
& =f\left(x_{0}\right)+\varepsilon x_{1} f^{\prime}\left(x_{0}\right)+p^{2}\left[x_{2} f^{\prime}\left(x_{0}\right)+\frac{1}{2} x_{1}^{2} f^{\prime \prime}\left(x_{0}\right)\right]+O\left(p^{3}\right)
\end{aligned}
$$

where $f^{\prime}(x)=\mathrm{d} f(x) / \mathrm{d} x$.

The solution of Eq. (92) is

$$
x_{0}(t)=A \cos \omega t
$$

Substitution of this result into the right side of Eq. (93) gives

$$
x_{1}^{\prime \prime}+\omega^{2} x_{1}=\left(1+\alpha_{1}\right) A \cos \omega t-\frac{A^{3} \cos ^{3} \omega t}{1+A^{2} \cos ^{2} \omega t}
$$

It is possible to do the following Fourier series expansion

$$
\frac{A^{3} \cos ^{3} \omega t}{1+A^{2} \cos ^{2} \omega t}=\sum_{n=0}^{\infty} a_{2 n+1} \cos [(2 n+1) \omega t]=a_{1} \cos \omega t+a_{3} \cos 3 \omega t+\ldots
$$

where 


$$
a_{2 n+1}=\frac{4}{\pi} \int_{0}^{\pi / 2} \frac{A^{3} \cos ^{3} \tau}{1+A^{2} \cos ^{2} \tau} \cos [(2 n+1) \tau] \mathrm{d} \tau
$$

and the first term of this expansion can be obtained by means of the following equation

$$
a_{1}=\frac{4}{\pi} \int_{0}^{\pi / 2} \frac{A^{3} \cos ^{3} \tau}{1+A^{2} \cos ^{2} \tau} \cos \tau \mathrm{d} \tau=A+\frac{2}{A}\left(\frac{1}{\sqrt{1+A^{2}}}-1\right)
$$

where $\tau=\omega t$. Substituting Eq. (98) into Eq. (97), we have

$$
x_{1}^{\prime \prime}+\omega^{2} x_{1}=\left[\left(1+\alpha_{1}\right) A-a_{1}\right] \cos \omega t-\sum_{n=1}^{\infty} a_{2 n+1} \cos [(2 n+1) \omega t]
$$

No secular terms in $x_{1}(t)$ requires eliminating contributions proportional to $\cos \omega t$ on the right-hand side of Eq. (101)

$$
\left(1+\alpha_{1}\right) A-a_{1}=0
$$

and we can easily find that the solution $\alpha_{1}$ is

$$
\alpha_{1}=\frac{a_{1}}{A}-1
$$

From Eqs. (12) and (103), writing $p=1$, we can easily find that the first order approximate frequency is

$$
\omega_{1}(A)=\sqrt{\frac{a_{1}}{A}}
$$


and substituting Eq. (100) into Eq. (104) we obtain

$$
\omega_{1}(A)=\sqrt{1+\frac{2}{A^{2}}\left(\frac{1}{\sqrt{1+A^{2}}}-1\right)}
$$

Now in order to obtain the correction term $x_{1}$ for the periodic solution $x_{0}$ we consider the following procedure. Taking into account Eqs. (101) and (102), we re-write Eq. (20) in the form

$$
x_{1}^{\prime \prime}+\omega^{2} x_{1}=-\sum_{n=1}^{\infty} a_{2 n+1} \cos [(2 n+1) \omega t]
$$

with initial conditions $x_{1}(0)=0$ and $x_{1}^{\prime}(0)=0$. The periodic solution to Eq. (106) can be written

$$
x_{1}(t)=\sum_{n=0}^{\infty} c_{2 n+1} \cos [(2 n+1) \omega t]
$$

Substituting Eq. (107) into Eq. (106) gives

$$
-\omega^{2} \sum_{n=0}^{\infty} 4 n(n+1) c_{2 n+1} \cos [(2 n+1) \omega t]=-\sum_{n=1}^{\infty} a_{2 n+1} \cos [(2 n+1) \omega t]
$$

and then we can write the following expression for the coefficients $c_{2 n+1}$

$$
c_{2 n+1}=\frac{a_{2 n+1}}{4 n(n+1) \omega^{2}}
$$

for $n \geq 1$. Taking into account that $x_{1}(0)=0$, Eq. (107) gives 


$$
c_{1}=-\sum_{n=1}^{\infty} c_{2 n+1}
$$

To determine the second-order approximate solution it is necessary to substitute Eq. (107) into Eq. (93). Then secular terms are eliminated and parameter $\alpha_{2}$ can be calculated. However, it is difficult to solve the new differential equation because, as $x_{1}(\mathrm{t})$ has a infinite number of harmonics, it would be necessary to multiply this infinite series by $\left(3+x_{0}^{2}\right) x_{0}^{2}\left(1+x_{0}^{2}\right)^{-2}$. At this moment we introduce a modification in He's homotopy perturbation method to simplify the solution procedure. $x_{1}(t)$ has an infinite number of harmonics, however we can truncate the series expansion at Eq. (107) and write an approximate equation $x_{1}^{(N)}(t)$ in the form

$$
x_{1}^{(N)}=\sum_{n=0}^{N} c_{2 n+1} \cos [(2 n+1) \omega t] \quad \text { and } \quad c_{1}=-\sum_{n=1}^{N} c_{2 n+1}
$$

which has only a finite number of harmonics. Comparing Eqs. (107) and (111), it follows that

$$
\lim _{N \rightarrow \infty} x_{1}^{(N)}(t)=x_{1}(t)
$$

In the simplest case we consider $N=1(n=0,1)$ in Eq. (111) and Eq. (110) becomes

$$
x_{1}^{(1)}(t)=c_{3}(\cos 3 \omega t-\cos \omega t)
$$

which has a similar form than the second order approximate solution considered in harmonic balance methods $[15,17]$. It is possible to do this approximation because the absolute value of the coefficient $c_{2 n+1}$ decreases when $n$ increase as we can easily verify from Eq. (109). From Eq. (109) the following expression for the coefficient $c_{3}$ is obtained 


$$
c_{3}=\frac{a_{3}}{8 \omega^{2}}
$$

where, from Eq. (99) we obtain

$$
a_{3}=\frac{4}{\pi} \int_{0}^{\pi / 2} \frac{A^{3} \cos ^{3} \tau}{1+A^{2} \cos ^{2} \tau} \cos 3 \tau \mathrm{d} \tau=\frac{2}{A}\left(4-\frac{3}{\sqrt{1+A^{2}}}\right)+\frac{8}{A^{3}}\left(1-\frac{1}{\sqrt{1+A^{2}}}\right)
$$

Substitution of Eq. (113) into Eq. (94) gives the following equation for $x_{2}(t)$

$$
x_{2}^{\prime \prime}+\omega^{2} x_{2}=\alpha_{2} x_{0}+\left(1+\alpha_{1}\right) x_{1}^{(1)}-\frac{\left(3+x_{0}^{2}\right) x_{0}^{2} x_{1}^{(1)}}{\left(1+x_{0}^{2}\right)^{2}}
$$

and taking into account Eqs. (96), (103), (113) and (114), Eq. (116) becomes

$$
\begin{aligned}
x_{2}^{\prime \prime} & +\omega^{2} x_{2}= \\
& =\alpha_{2} A \cos \omega t+\frac{a_{1} a_{3}(\cos 3 \omega t-\cos \omega t)}{8 A \omega^{2}}-\frac{a_{3} A^{2}\left(3+A^{2} \cos ^{2} \omega t\right)(\cos 3 \omega t-\cos \omega t) \cos ^{2} \omega t}{8 \omega^{2}\left(1+A^{2} \cos ^{2} \omega t\right)^{2}}
\end{aligned}
$$

It is possible to do the following Fourier series expansion

$$
\begin{aligned}
& \frac{A^{2}\left(3+A^{2} \cos ^{2} \omega t\right)(\cos 3 \omega t-\cos \omega t) \cos ^{2} \omega t}{\left(1+A^{2} \cos ^{2} \omega t\right)^{2}}= \\
& \quad=\sum_{n=0}^{\infty} b_{2 n+1} \cos [(2 n+1) \omega t]=b_{1} \cos \omega t+b_{3} \cos 3 \omega t+\ldots
\end{aligned}
$$

where the first term of this expansion can be obtained by means of the following equation 


$$
\begin{aligned}
b_{1}=\frac{4}{\pi} \int_{0}^{\pi / 2} \frac{A^{2}\left(3+A^{2} \cos ^{2} \tau\right)(\cos 3 \tau-\cos \tau) \cos ^{2} \tau}{\left(1+A^{2} \cos ^{2} \tau\right)^{2}} \cos \tau \mathrm{d} \tau= \\
=-1+\frac{4}{A^{2}}\left(1+\frac{4}{\sqrt{1+A^{2}}}\right)-\frac{24}{A^{4}}\left(1-\frac{1}{\sqrt{1+A^{2}}}\right)
\end{aligned}
$$

From Eqs. (117) and (118), the secular term in the solution for $x_{2}(t)$ can be eliminated if

$$
\alpha_{2} A-\frac{a_{1} a_{3}}{8 A \omega^{2}}-\frac{a_{3} b_{1}}{8 \omega^{2}}=0
$$

Equation (119) can be solved for $\alpha_{2}$ and we obtain

$$
\alpha_{2}=\frac{a_{1} a_{3}}{8 A^{2} \omega^{2}}+\frac{a_{3} b_{1}}{8 A \omega^{2}}
$$

From Eqs. (12), (100), (115) and (119), and taking $p=1$, one can easily obtain the following expression for the second-order approximate frequency is

$$
\omega_{2}(A)=\sqrt{\frac{a_{1}}{2 A}+\sqrt{\frac{a_{1}^{2}}{4 A^{2}}+\frac{a_{1} a_{3}}{8 A^{2}}+\frac{a_{3} b_{1}}{8 A}}}
$$

With the requirement of Eq. (120), we can re-write Eq. (117) in the form

$$
x_{2}^{\prime \prime}+\omega^{2} x_{2}=\frac{a_{1} a_{3} \cos 3 \omega t}{8 A \omega^{2}}-\frac{a_{3}}{8 \omega^{2}} \sum_{n=1}^{\infty} b_{2 n+1} \cos [(2 n+1) \omega t]
$$

with initial conditions $x_{2}(0)=0$ and $x_{2}^{\prime}(0)=0$. The general solution of this equation is 


$$
x_{2}(t)=\sum_{n=0}^{\infty} d_{2 n+1} \cos [(2 n+1) \omega t]
$$

Substituting Eq. (124) into Eq. (123) gives

$-\omega^{2} \sum_{n=0}^{\infty} 4 n(n+1) d_{2 n+1} \cos [(2 n+1) \omega t]=\frac{a_{1} a_{3} \cos 3 \omega t}{8 A \omega^{2}}-\frac{a_{3}}{8 \omega^{2}} \sum_{n=1}^{\infty} b_{2 n+1} \cos [(2 n+1) \omega t]$

and then we can write the following expression for the coefficients $d_{2 n+1}$

$$
\begin{gathered}
d_{3}=-\frac{a_{1} a_{3}}{64 A \omega^{2}}+\frac{a_{3} b_{3}}{64 \omega^{2}} \\
d_{2 n+1}=\frac{a_{3} b_{2 n+1}}{32 n(n+1) \omega^{4}} \text { for } n \geq 2
\end{gathered}
$$

where $\omega$ is given by Eq. (122) and $b_{3}$ can be calculated as follows

$$
\begin{gathered}
b_{3}=\frac{4}{\pi} \int_{0}^{\pi / 2} \frac{A^{2}\left(3+A^{2} \cos ^{2} \tau\right)(\cos 3 \tau-\cos \tau) \cos ^{2} \tau}{\left(1+A^{2} \cos ^{2} \tau\right)^{2}} \cos 3 \tau \mathrm{d} \tau= \\
=1+\frac{8}{A^{2}}\left(1-\frac{6}{\sqrt{1+A^{2}}}\right)+\frac{40}{A^{4}}\left(3-\frac{5}{\sqrt{1+A^{2}}}\right)+\frac{160}{A^{6}}\left(1-\frac{1}{\sqrt{1+A^{2}}}\right)
\end{gathered}
$$

Taking into account that $x_{2}(0)=0$, Eq. (124) gives

$$
d_{1}=-\sum_{n=1}^{\infty} d_{2 n+1}
$$


and truncating the infinite series at Eq. (124), it is possible to obtain the following secondorder approximate solution for $x_{2}$

$$
x_{2}^{(N)}(t)=\sum_{n=0}^{N} d_{2 n+1} \cos [(2 n+1) \omega t] \quad \text { and } \quad d_{1}=-\sum_{n=1}^{N} d_{2 n+1}
$$

which has only a finite number of harmonics. Comparing Eqs. (124) and (130), it follows that

$$
\lim _{N \rightarrow \infty} x_{2}^{(N)}(t)=x_{2}(t)
$$

As we are analyzing the second-order approximation we consider $N=2$ in Eq. (131), in other words, only three harmonics $(n=0,1,2)$. In this situation, it is easy to verify that

$$
d_{5}=\frac{a_{3} b_{5}}{192 \omega^{4}}
$$

where $\omega$ is given by Eq. (122) and $b_{5}$ can be calculated as follows

$$
\begin{aligned}
b_{5}= & \frac{4}{\pi} \int_{0}^{\pi / 2} \frac{A^{2}\left(3+A^{2} \cos ^{2} \tau\right)(\cos 3 \tau-\cos \tau) \cos ^{2} \tau}{\left(1+A^{2} \cos ^{2} \tau\right)^{2}} \cos 5 \tau \mathrm{d} \tau= \\
& =-\frac{8}{A^{2}}\left(1-\frac{1}{\sqrt{1+A^{2}}}\right)-\frac{8}{A^{4}}\left(39-\frac{795}{\sqrt{1+A^{2}}}\right)-\frac{224}{A^{6}}\left(5-\frac{7}{\sqrt{1+A^{2}}}\right)-\frac{896}{A^{8}}\left(1-\frac{1}{\sqrt{1+A^{2}}}\right)
\end{aligned}
$$

From Eq. (130) we obtain the following value for $d_{1}$

$$
d_{1}=-\sum_{n=1}^{2} d_{2 n+1}=-d_{3}-d_{5}
$$


Taking this into account, $x_{2}^{(2)}$ can be written as follows

$$
x_{2}^{(2)}(t)=d_{3}(\cos 3 \omega t-\cos \omega t)+d_{5}(\cos 5 \omega t-\cos \omega t)
$$

From Eqs. (11), (96), (113) and (135), and taking $p=1$, one can easily obtain the following expression for the second-order approximate solution

$$
x(t)=x_{0}(t)+x_{1}^{(1)}(t)+x_{2}^{(2)}(t)=\left(A-c_{3}-d_{3}-d_{5}\right) \cos \omega t+\left(c_{3}+d_{3}\right) \cos 3 \omega t+d_{5} \cos 5 \omega t
$$

which has a similar form to the third-order approximate solution considered in harmonic balance methods.

\section{2.- Comparison with the exact and other approximate solution}

We illustrate the accuracy of the modified approach by comparing the approximate solutions previously obtained with the exact frequency $\omega_{e}$ and other results in the literature. In particular, we will consider the solution of Eq. (84) using the harmonic balance method applied to Eq. (84) [16]. This method is a procedure for determining analytical approximations to the periodic solutions of differential equations using a truncated Fourier series representation. Like the homotopy perturbation method, the harmonic balance method can be applied to nonlinear oscillatory problems where a linear term does not exist, the nonlinear terms are not small, and there is no perturbation parameter. However, it is very difficult to use the harmonic balance method to construct higher-order analytical approximations because this method requires solving analytical solutions of sets of algebraic equations with very complex nonlinearities.

Calculation of the exact angular frequency, $\omega_{e}(A)$, proceeds as follows. By integrating Eq. (84) and using the initial conditions in Eq. (85), we arrive at 


$$
\frac{1}{2}\left(\frac{\mathrm{d} x}{\mathrm{~d} t}\right)^{2}+\frac{1}{2} x^{2}-\frac{1}{2} \log \left(1+x^{2}\right)=\frac{1}{2} A^{2}-\frac{1}{2} \log \left(1+A^{2}\right)
$$

From the representation above, we can derive the exact frequency as follows

$$
\omega_{e}(A)=\frac{\pi}{2}\left[A \int_{0}^{1} \frac{\mathrm{d} u}{\sqrt{A^{2}\left(1-u^{2}\right)+\log \left[\left(1+A^{2} u^{2}\right) /\left(1+A^{2}\right)\right]}}\right]^{-1}
$$

Lim and $\mathrm{Wu}$ [16] approximately solved Eq. (84) by using an improved harmonic balance method in which linearization is carried out prior to harmonic balancing. They achieved the following results for the first and the second approximation orders

$$
\begin{gathered}
\omega_{L W 1}(A)=\sqrt{1+\frac{2}{A^{2}}\left(\frac{1}{\sqrt{1+A^{2}}}-1\right)} \\
\omega_{L W 2}(A)=\sqrt{g(A)+\sqrt{g^{2}(A)-h(A)}}
\end{gathered}
$$

where the expressions for $g(A)$ and $h(A)$ are given in the Appendix. From Eqs. (105) and (139), we can see that the first-order homotopy perturbation method and the first-order harmonic balance method give the same result for the approximate frequency.

For small values of the amplitude $A$ it is possible to take into account the following power series expansions

$$
\omega_{e}(A)=\frac{\pi}{2 \sqrt{2} K(-1)} A-\ldots=0.847213 A-\ldots
$$




$$
\begin{gathered}
\omega_{2}(A)=\frac{1}{4} \sqrt{6+\sqrt{30}} A-\ldots=0.846951 A-\ldots \\
\omega_{L W 2}(A)=\frac{1}{6} \sqrt{\frac{31+\sqrt{421}}{2}} A-\ldots=0.845891 A-\ldots
\end{gathered}
$$

In Eq. (141), $K(m)$ is the complete elliptical integral of the first kind defined in equation (22) and $K(-1)=1.31103$. If we compare the first term in the expansion of the angular frequency $\omega_{2}(A)\left(\right.$ Eq. (142)) and in the expansion of the angular frequency $\omega_{L W 2}(A)$ (Eq. (143)) with the first term in the series expansion of the exact angular frequency $\omega_{e}$ (Eq. (141)), we can see that the relative errors in the first term in the series expansions of $\omega_{2}$ and $\omega_{L W 2}$ are $0.031 \%$ and $0.16 \%$, respectively. This implies that the second-order approximate angular frequency $\omega_{2}(A)$ obtained in this paper provides excellent approximations to the exact frequency $\omega_{e}(A)$.

For very large values of the amplitude $A$ it is possible to take into account the following power series expansions

$$
\begin{gathered}
\omega_{e}(A)=1-\frac{1}{A^{2}}+\ldots \\
\omega_{2}(A)=1-\frac{1}{A^{2}}+\ldots \\
\omega_{L W 2}(A)=1-\frac{1}{A^{2}}+\ldots
\end{gathered}
$$

Furthermore, we have the following equations

$$
\lim _{A \rightarrow 0} \frac{\omega_{2}(A)}{\omega_{e}(A)}=0.999691
$$




$$
\begin{gathered}
\lim _{A \rightarrow 0} \frac{\omega_{L W 2}(A)}{\omega_{e}(A)}=0.99844 \\
\lim _{A \rightarrow \infty} \frac{\omega_{2}(A)}{\omega_{e}(A)}=1 \\
\lim _{A \rightarrow \infty} \frac{\omega_{L W 2}(A)}{\omega_{e}(A)}=1
\end{gathered}
$$

In Figure 8 we plotted the relative errors for the approximate frequencies $\omega_{2}(A)$ (Eq. (122)) and $\omega_{L W 2}(A)$ (Eq. (140)). In this figure the percentage errors were calculated using the following equation

$$
\text { Relative error of } \omega_{j}(\%)=100\left|\frac{\omega_{j}-\omega_{e}}{\omega_{e}}\right| \quad j=2, L W 2
$$

As we can see from Figure 8, the relative errors for $\omega_{2}(A)$ are lower than $0.078 \%$ (value for $A=0.9870$ ) for all the range of values of amplitude of oscillation $A$, and these relative errors tend to $0.031 \%$ when $A$ tends to zero and tend to zero when $A$ tends to infinity (see Eqs. (147) and (149)). However, the relative errors for $\omega_{L W 2}(A)$ are lower than $0.17 \%$ (value for $A=0.5960$ ) for all the range of values of amplitude of oscillation $A$, and these relative errors tend to $0.16 \%$ when $A$ tends to zero and tend to zero when $A$ tends to infinity (see Eqs. (148) and (150)). Figure 8 indicates that $\omega_{2}(A)$ provides excellent approximations to the exact frequency $\omega_{e}(A)$ for the range of values of oscillation amplitude.

The exact periodic solutions $x(t)$ achieved by numerically integrating Eq. (84), and the proposed normalized second-order approximate periodic solution $x(t)$ in Eq. (136) for one complete cycle are plotted in Figure 9 for oscillation amplitude $A=0.9870$ (value of $A$ for which the relative error for the approximate frequency $\omega_{2}$ is maximum). In this 
figure parameter $h$ is defined in equation (74). Figure 9 shows that Eq. (136) provides a good approximation to the exact periodic solution. The results presented here illustrated very good agreement of the second-order approximate frequency obtained in this paper, $\omega_{2}(A)$, with the exact frequency $\omega_{e}(A)$. It is clear that at the second approximation order, the result obtained in this paper is better than those obtained previously by other authors.

\section{Conclusions}

The homotopy perturbation method has been used to obtain two approximate frequencies for a relativistic oscillatory system. An approximation to the periodic solutions was calculated by transforming to a new set of variables, $(x, y) \rightarrow(x, u)$, and the homotopy perturbation method was applied. Excellent agreement between approximate frequencies and the exact one has been demonstrated and discussed, and the discrepancy of the second-order approximate frequency, $\omega_{2}^{\prime}(A)$, with respect to the exact one is as low as $0.18 \%$. The power series expansions of the exact and approximate frequencies were also compared for low as well as large values of the oscillation amplitudes. Some examples were presented to illustrate the excellent accuracy of the approximate analytical solutions. The homotopy perturbation method has been also used to obtain the second order approximate frequency for the Duffing-harmonic oscillator. Although the lowest order homotopy perturbation method approximation to the Duffing-harmonic oscillator [34] is very good for all values of oscillation amplitude (Eq. (105)), the second analytical approximation derived here is even better. Excellent agreement between the second-order approximate frequency, $\omega_{2}(A)$, and the exact one has been demonstrated and discussed, and the discrepancy of this second-order approximate frequency with respect to the exact one is as low as $0.078 \%$ and tends to $0.031 \%$ when $A$ tends to zero and the discrepancy tends to $0 \%$ when $A$ tends to infinity. Finally, we can see that the method considered here is very simple in its principle, and very easy to apply, and we think that the method has great potential and can be applied to other strongly nonlinear oscillators with nonpolynomial terms.

\section{Appendix}


The second-order approximate frequency obtained by Lim and $\mathrm{Wu}$ [16] applying the harmonic balance method to Eq. (84) is given in Eq. (126)

$$
\omega_{L W 2}(A)=\sqrt{g(A)+\sqrt{g^{2}(A)-h(A)}}
$$

where

$$
\begin{aligned}
& g(A)=\frac{\left(b_{0}-b_{2}-b_{4}+b_{6}\right) A+18 a_{1}+2 a_{3}}{36 A} \\
& h(A)=\frac{a_{1}\left(b_{0}-b_{2}-b_{4}+b_{6}\right)+a_{3}\left(b_{0}-b_{4}\right)}{18 A}
\end{aligned}
$$

where $a_{1}$ and $a_{3}$ are given in Eqs. (100) and (115), respectively, and

$$
\begin{gathered}
b_{0}=2+\frac{2}{\sqrt{1+A^{2}}}-\frac{2\left(2+A^{2}\right)}{\left(1+A^{2}\right)^{3 / 2}} \\
b_{2}=\frac{4}{A^{2}}-\frac{2}{\sqrt{1+A^{2}}}-\frac{4}{A^{2} \sqrt{1+A^{2}}}+\frac{2 A^{2}}{\left(1+A^{2}\right)^{3 / 2}} \\
b_{4}=\frac{16}{A^{4}}\left[-3-\frac{1}{2} A^{2}+\frac{8+8 A^{2}+A^{4}}{\left.8 \sqrt{1+A^{2}}+\frac{16+24 A^{2}+6 A^{4}-A^{6}}{8\left(1+A^{2}\right)^{3 / 2}}\right]}\right. \\
b_{6}=\frac{16}{A^{4}}\left[\frac{20}{A^{2}}+12+\frac{3}{4} A^{2}+\frac{48+22 A^{2}+3 A^{4}}{8 \sqrt{1+A^{2}}}+\frac{4}{A^{2} \sqrt{1+A^{2}}}-\frac{192+416 A^{2}+280 A^{4}+60 A^{6}+3 A^{8}}{8 A^{2}\left(1+A^{2}\right)^{3 / 2}}\right.
\end{gathered}
$$




\section{Acknowledgements}

This work was supported by the "Ministerio de Educación y Ciencia", Spain, under project FIS2005-05881-C02-02 and the "Generalitat Valenciana", Spain, under project ACOMP/ 2007/020. 


\section{References}

[1] Nayfeh A H 1985 Problems in Perturbations (Wiley, New York)

[2] He J H 2001 Modified Lindstedt-Poincare methods for some non-linear oscillations. Part III: double series expansion Int. J. Nonlinear Sci. Numer. Simul. 2 317-20

[3] He J H 2002 Modified Lindstedt-Poincare methods for some non-linear oscillations. Part I: expansion of a constant Int. J. Nonlinear Mech. 37 309-14

[4] He J H 2002 Modified Lindstedt-Poincare methods for some non-linear oscillations. Part II: a new transformation Int. J. Nonlinear Mech. 37 315-20

[5] Amore P and Fernández F M 2005 Exact and approximate expressions for the period of anharmonic oscillators Eur. J. Phys. 26 589-601

[6] Amore P, Raya A and Fernández F M 2005 Alternative perturbation approaches in classical mechanics Eur. J. Phys. 26 1057-63

[7] He J H and Wu X H 2006 Construction of solitary solution and compact on-like solution by variational iteration method Chaos Solitons Fractals 29 108-13

[8] He J H 2007 Variational approach for nonlinear oscillators Chaos Solitons Fractals 34 1430-9

[9] Dehghan M and Tatari M 2007 The use of He's variational iteration method for solving multipoint boundary value problems Phys. Scr. 72 672-6

[10] Tatari M and Dehghan M 2006 The use of He's variational iteration method for solving a Fokker-Planck equation Phys. Scr. 74 310-6 
[11] Tatari M and Dehghan M 2007 On the convergence of He's variational iteration method J. Comput. Appl. Math. 207 121-8

[12] Tatari M and Dehghan M 2007 He's variational iteration method for computing a control parameter in a semi-linear inverse parabolic equation Chaos Solitons Fractals 33 671-7

[13] Tatari M and Dehghan M 2007 Solution of problems in calculus of variations via He's variational iteration method Phys. Lett. A 362 401-6

[14] Mickens R E 1996 Oscillations in Planar Dynamics Systems (World Scientific, Singapore)

[15] Mickens R E 1984 Comments on the method of harmonic-balance J. Sound. Vib. 94 456-60

[16] Lim C W, Wu B S and Sun W P 2006 Higher accuracy analytical approximations to the Duffing-harmonic oscillator J. Sound Vib. 296 1039-45

[17] Beléndez A, Hernández A, Márquez A, Beléndez T and Neipp C 2006 Analytical approximations for the period of a simple pendulum Eur. J. Phys. 27 539-51

[18] Beléndez A, Pascual C 2007 Harmonic balance approach to the periodic solutions of the (an)harmonic relativistic oscillator Phys. Lett. A 371 291-9

[19] Beléndez A, Hernández A, Beléndez T, Álvarez M L, Gallego S, Ortuño M and Neipp C 2007 Application of the harmonic balance method to a nonlinear oscillator typified by a mass attached to a stretched wire J. Sound Vib. 302 1018-29

[20] Chowdhury M S H and Hashim I 2007 Application of homotopy-perturbation method to nonlinear population dynamics models Phys. Lett. A 368 251-8 
[21] Özis T and Yildirim A 2007 A comparative study of He's homotopy perturbation method for determining frequency-amplitude relation of a nonlinear oscillator with discontinuities Int. J. Nonlinear Sci. Numer. Simul. 8 243-8

[22] He J H 2006 Some asymptotic methods for strongly nonlinear equations Int. J. Mod. Phys. B 20 1141-99

[23] He J H 2006 Non-perturbative methods for strongly nonlinear problems (dissertation.de-Verlag im Internet $\mathrm{GmbH}$, Berlin)

[24] He J H 2006 New interpretation of homotopy perturbation method Int. J. Mod. Phys. B $202561-8$

[25] Dehghan M and Shakeri F 2008 Solution of an integro-differential equation arising in oscillating magnetic fields using He's homotopy perturbation method Progress in Electromagnetics Research 78 361-76

[26] Cai X C, Wu W Y and Li M S 2006 Approximate period solution for a kind of nonlinear oscillator by He's perturbation method Int. J. Nonlinear Sci. Numer. Simul. 7 109-17

[27] Chowdhury M S H and Hashim I 2007 Solutions of time-dependent Emden-Fowler type equations by homotopy-perturbation method Phys. Lett. A 368 305-13

[28] Beléndez A, Hernández A, Beléndez T, Fernández E, Álvarez M L and Neipp C 2007 Application of He's homotopy perturbation method to the Duffing-harmonic oscillator Int. J. Nonlinear Sci. Numer. Simul. 8 79-88

[29] Beléndez A, Hernández A, Beléndez T, Neipp C and Márquez A 2007 Application of the homotopy perturbation method to the nonlinear pendulum Eur. J. Phys. 28 93104 
[30] Ganji D D and Sadighi A 2006 Application of He's homotopy-perturbation method to nonlinear coupled systems of reaction-diffusion equations Int. J. Nonlinear Sci. Numer. Simul. 7 411-8

[31] Siddiqui A, Mahmood R and Ghori Q 2006 Thin film flow of a third grade fluid on moving a belt by He's homotopy perturbation method Int. J. Nonlinear Sci. Numer. Simul. 7 15-26

[32] Shakeri F and Dehghan M 2007 Solution of the delay differential equations via homotopy perturbation method Math. Comp. Mod. 46 1434-41

[33] Rafei M and Ganji D D 2006 Explicit solutions of Helmhotlz equation and fifthorder $\mathrm{KdV}$ equation using homotopy perturbation method Int. J. Nonlinear Sci. Numer. Simul. 7 321-8

[34] Ariel P D and Hayat T 2006 Homotopy perturbation method and axisymmetric flow over a stretching sheet Int. J. Nonlinear Sci. Numer. Simul. 7 399-406

[35] Özis T and Yildirim A 2007 A comparative study of He's homotopy perturbation method for determining frequency-amplitude relation of a nonlinear oscillator with discontinuities Int. J. Nonlinear Sci. Numer. Simul. 8 243-8

[36] Beléndez A, Pascual C, Gallego S, Ortuño M, Neipp C 2007 Application of a modified He's homotopy perturbation method to obtain higher-order approximations of a $x^{1 / 3}$ force nonlinear oscillator Phys. Lett. A $\mathbf{2 6}, 421-6$

[37] Shakeri F and Dehghan M 2007 Inverse problem of diffusion by He's homotopy perturbation method Phys. Scr. 75 551-6

[38] Dehghan M and Shakeri F 2007 Solution of a partial differential equation subject to temperature overspecification by He's homotopy perturbation method Phys. Scr. 75 778-87 
[39] Beléndez A, Pascual C, Méndez D, Márquez A 2007 Application of He’s Homotopy Perturbation Method to the relativistic (an)harmonic oscillator. I: Comparison between approximate and exact frequencies Int. J. Non-linear Sci. Numer. Simul. 8 (4) 483-91

[40] Beléndez A, Pascual C, Méndez D, Álvarez M L, Neipp C 2007 Application of He’s Homotopy Perturbation Method to the relativistic (an)harmonic oscillator. II: A More Accurate Approximate Solution Int. J. Non-linear Sci. Numer. Simul. 8 (4) 493-504

[41] Mickens R E 1998 Periodic solutions of the relativistic harmonic oscillator J. Sound Vib. 212, 905-8

[42] Milne-Thomson L M 1972 'Elliptic integrals' in Abramowitz M and Stegun I A (Eds) Handbook of Mathematical Functions (Dover Publications, Inc., New York)

[43] Moreau W, Easther R, Neutze R 1994 Relativistic (an)harmonic oscillator Am. J. Phys. 62, 531-5

[44] Mickens R E 2001 Mathematical and numerical study of the Duffing-harmonic oscillator J. Sound Vib. 244 563-7

[45] Beléndez A, Beléndez T, Márquez A and Neipp C Application of He's homotopy perturbation method to conservative truly nonlinear oscillators Chaos Solitons Fractals (2006), doi:10.1016/j.chaos.2006.09.070 


\section{FIGURE CAPTIONS}

Figure 1.- Relative error for approximate frequencies $\omega_{1}$ (dashed line) and $\omega_{2} \equiv \omega_{2}^{(1)}$ (continuous line) for the relativistic oscillator.

Figure 2.- Comparison of the analytical approximate solution ( $\circ$ ) with the exact solution (continuous line) for $A=1\left(\beta_{0}=v_{0} / c=0.74536\right)$ for the relativistic oscillator.

Figure 3.- Comparison of the analytical approximate solution (o) with the exact solution (continuous line) for $A=2\left(\beta_{0}=v_{0} / c=0.94281\right)$ for the relativistic oscillator.

Figure 4.- Comparison of the analytical approximate solution $\left({ }^{\circ}\right)$ with the exact solution (continuous line) for $A=10\left(\beta_{0}=v_{0} / c=0.99981\right)$ for the $\mathrm{v}$.

Figure 5.- Comparison of the analytical approximate solution (o) with the exact solution (continuous line) for $A=100\left(\beta_{0}=v_{0} / c=0.99999998\right)$ for the relativistic oscillator.

Figure 6.- Relative error for approximate frequencies $\omega_{2} \equiv \omega_{2}^{(1)}$ (dashed line) and $\omega_{2}^{\prime} \equiv \omega_{2}^{(2)}$ (continuous line) for the relativistic oscillator

Figure 7.- Comparison of the analytical approximate solution ( $\circ$ ) with the exact solution (continuous line) for $A=100100\left(\beta_{0}=v_{0} / c=0.99999998\right)$ when the approximate frequency $\omega_{2}^{\prime} \equiv \omega_{2}^{(2)}$ is considered (relativistic oscillator).

Figure 8.- Relative errors for second-order approximate frequencies $\omega_{L W 2}(A)$, Eq. (140) (dashed line) and $\omega_{2}(A)$, Eq. (122) (continuous line). For the doffing-harmonic oscillator. 
Figure 9.- Comparison of the second-order analytical approximate solution for the doffing-harmonic oscillator, Eq. (136) (dashed line and circles) with the numerical exact solution (continuous line) for $A=0.9870$. 
FIGURE 1

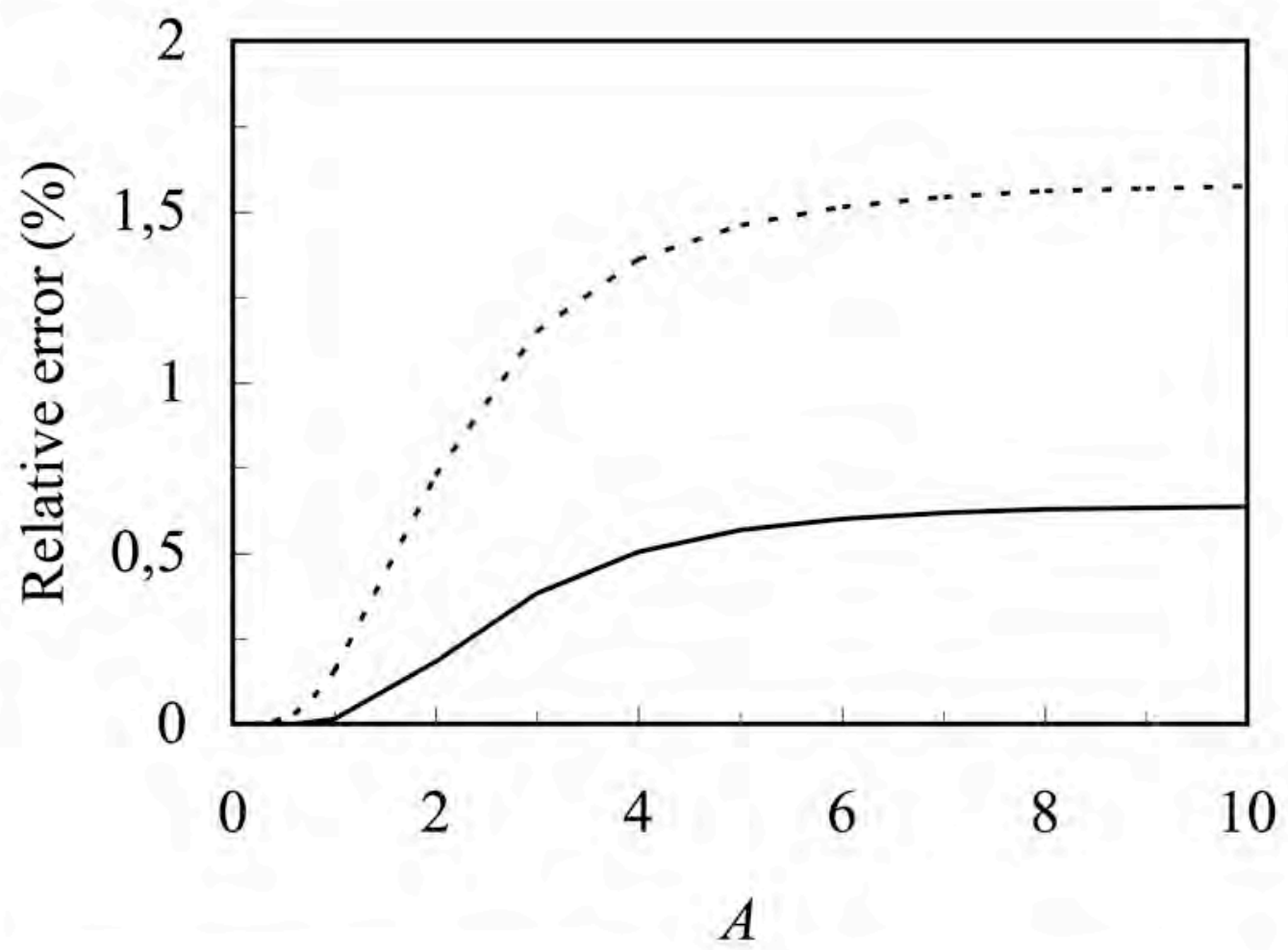


FIGURE 2

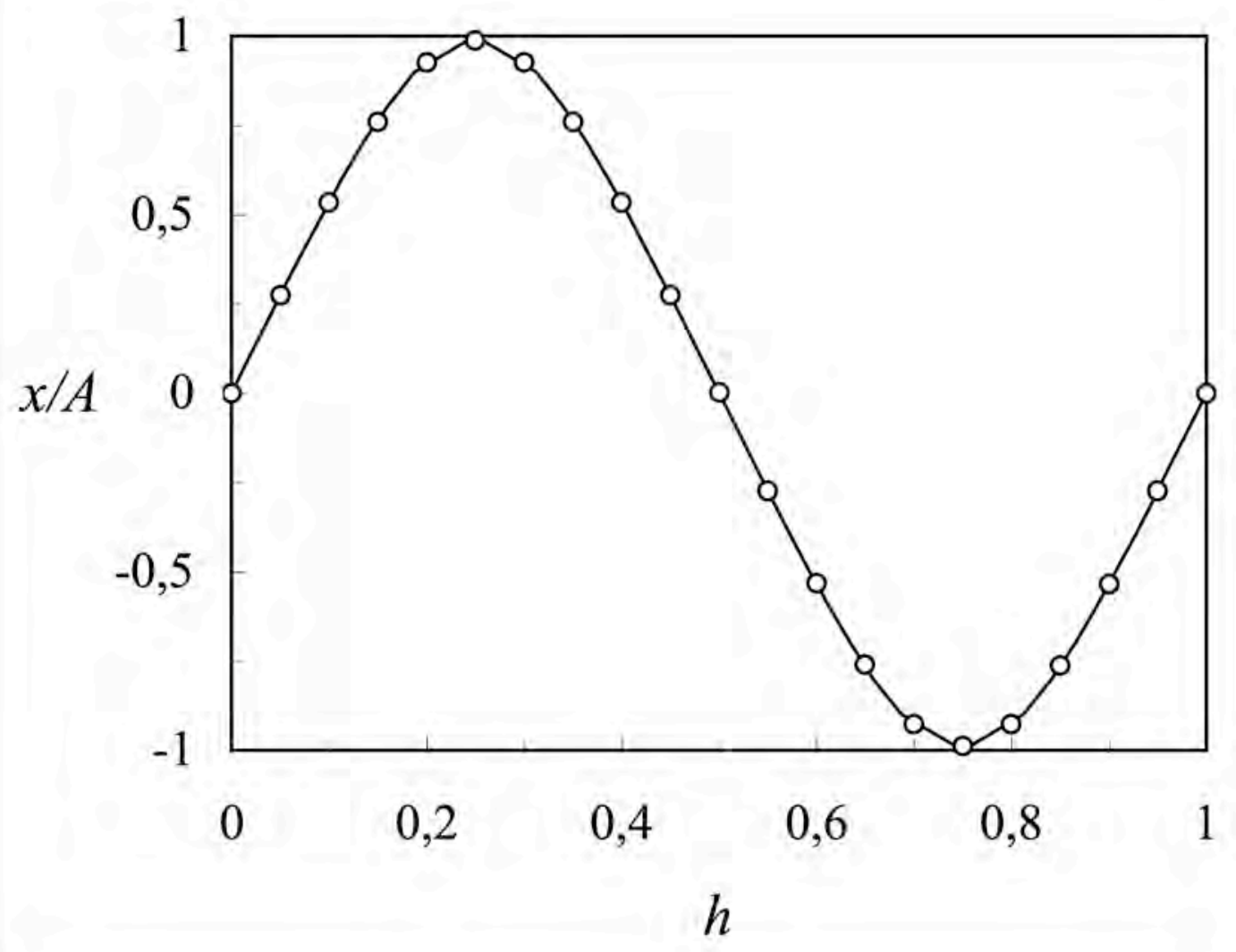


FIGURE 3

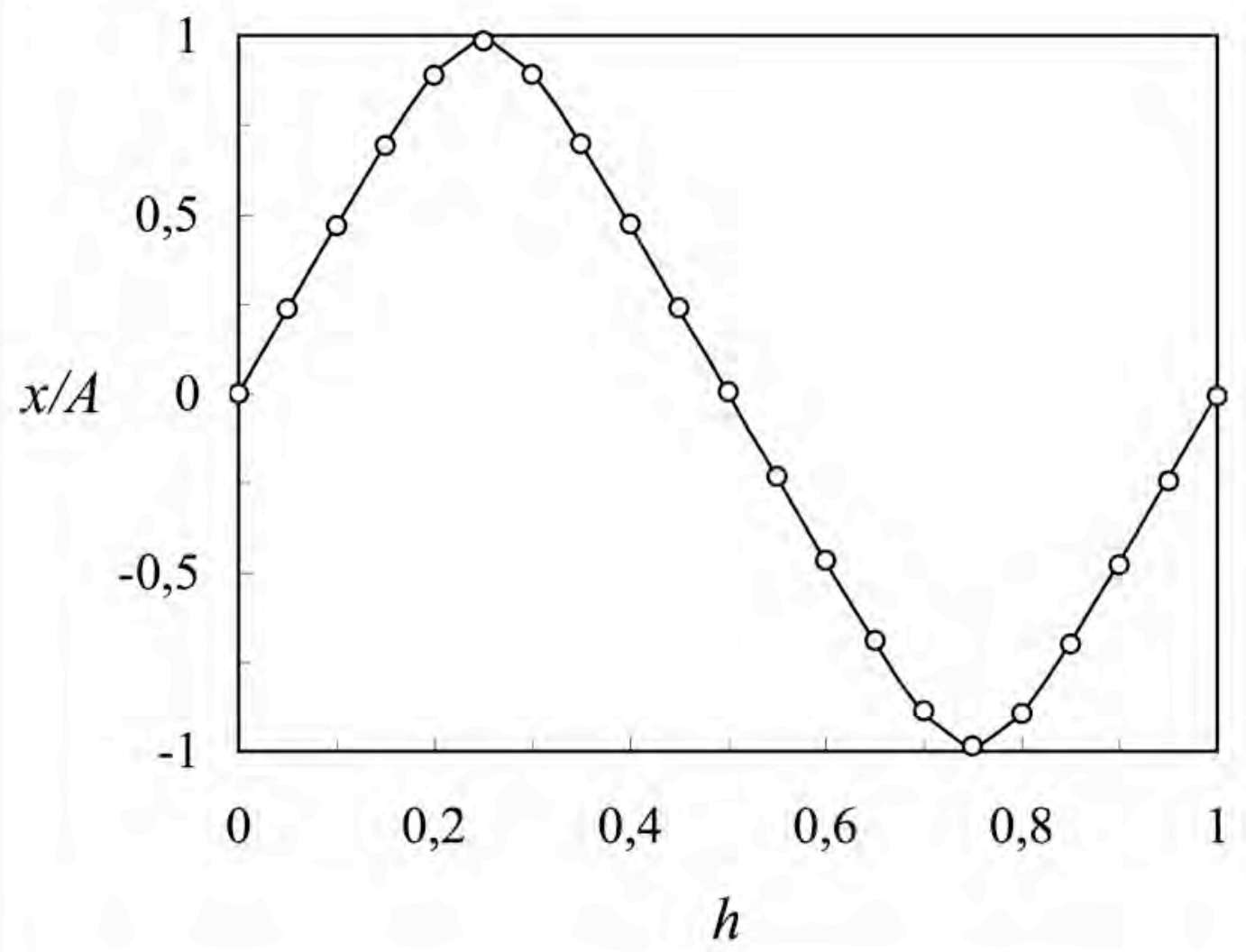


FIGURE 4

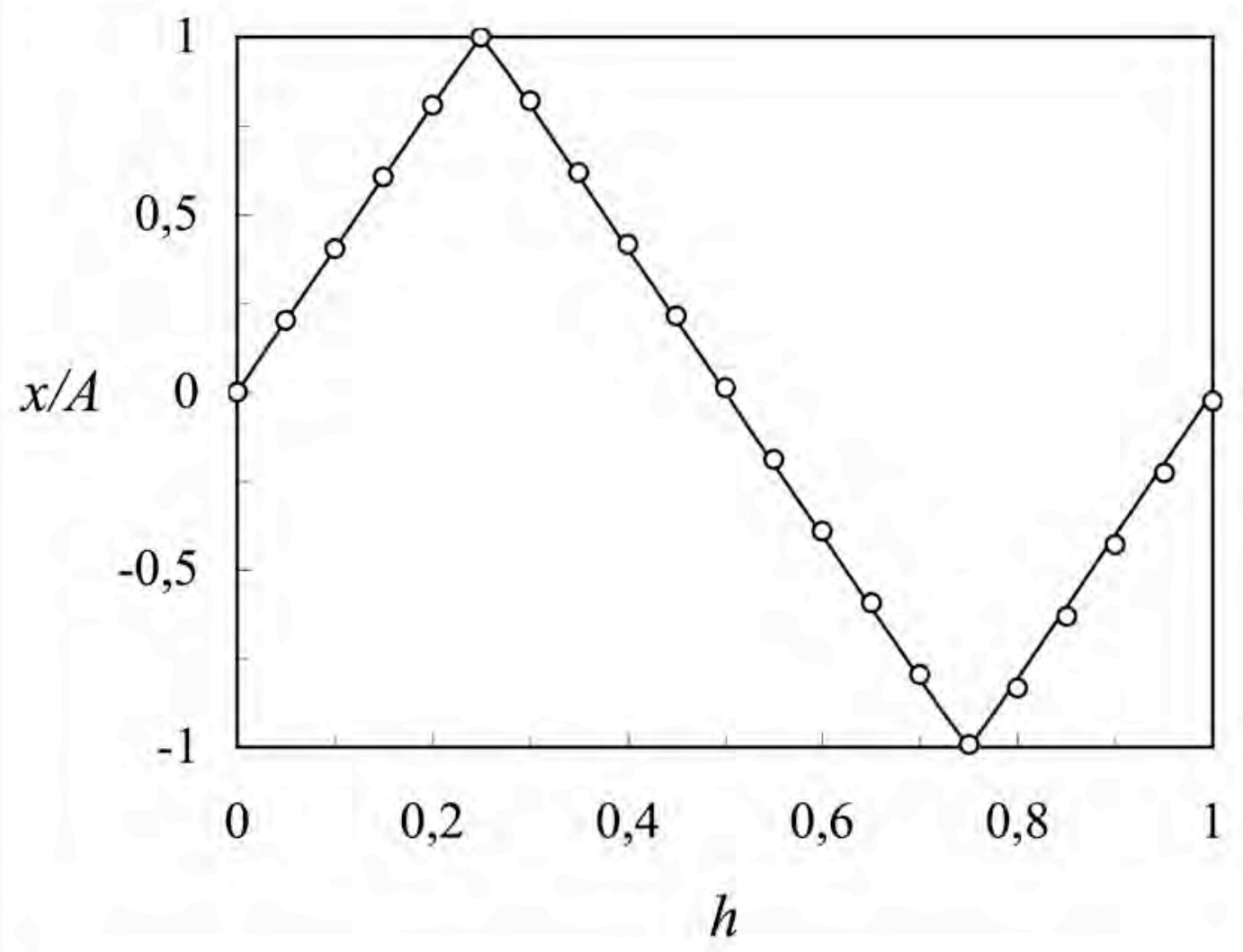


FIGURE 5

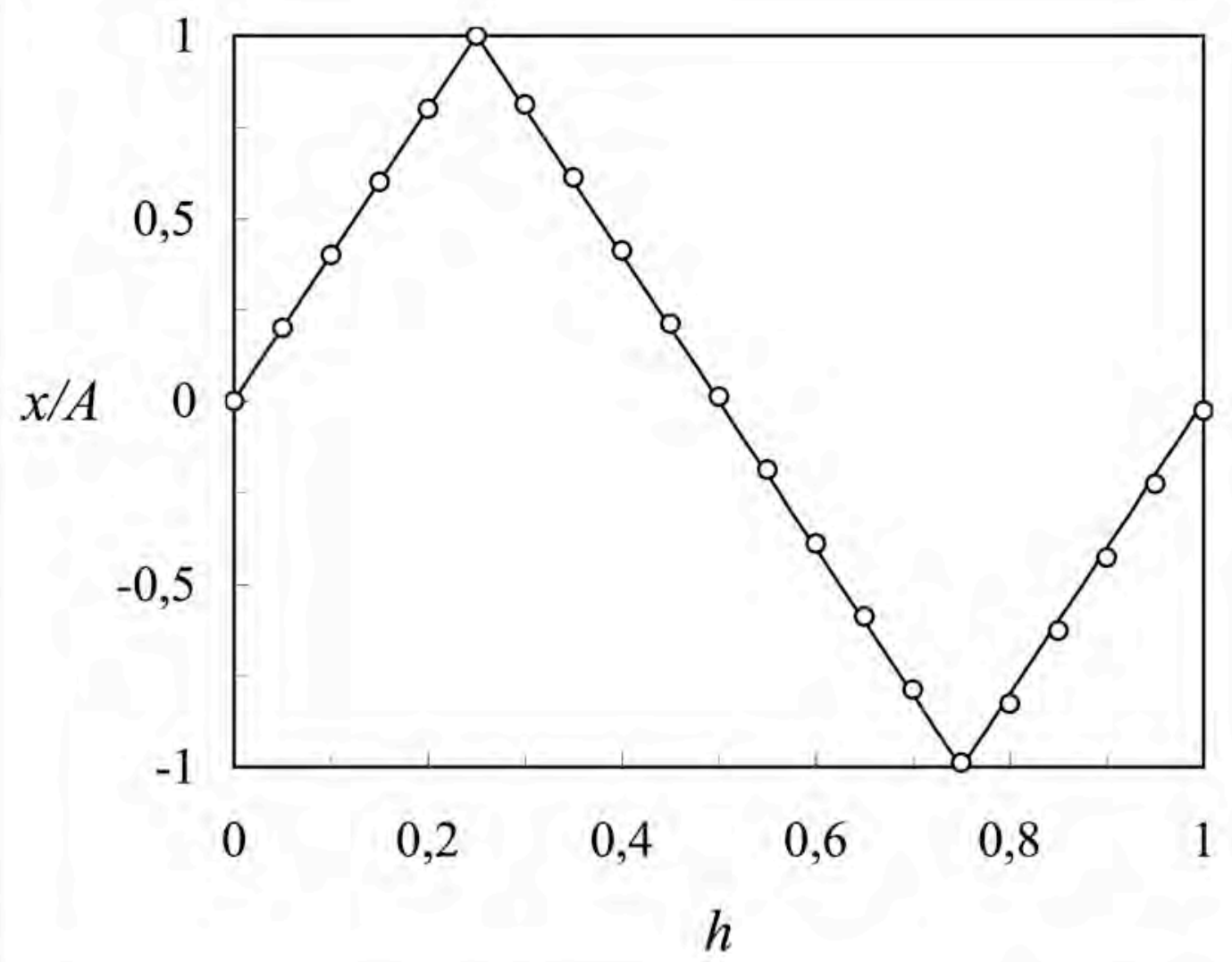


FIGURE 6

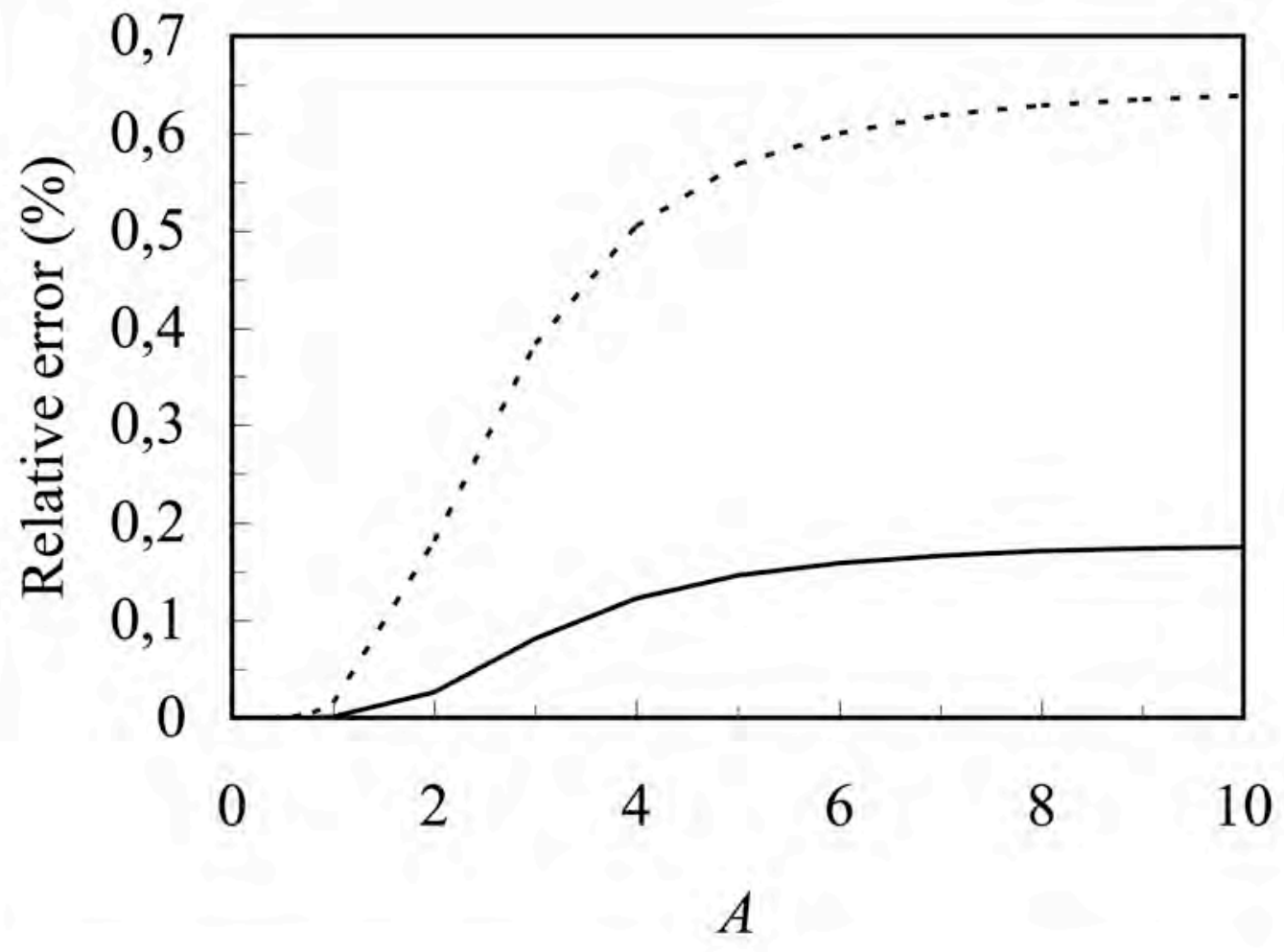


FIGURE 7

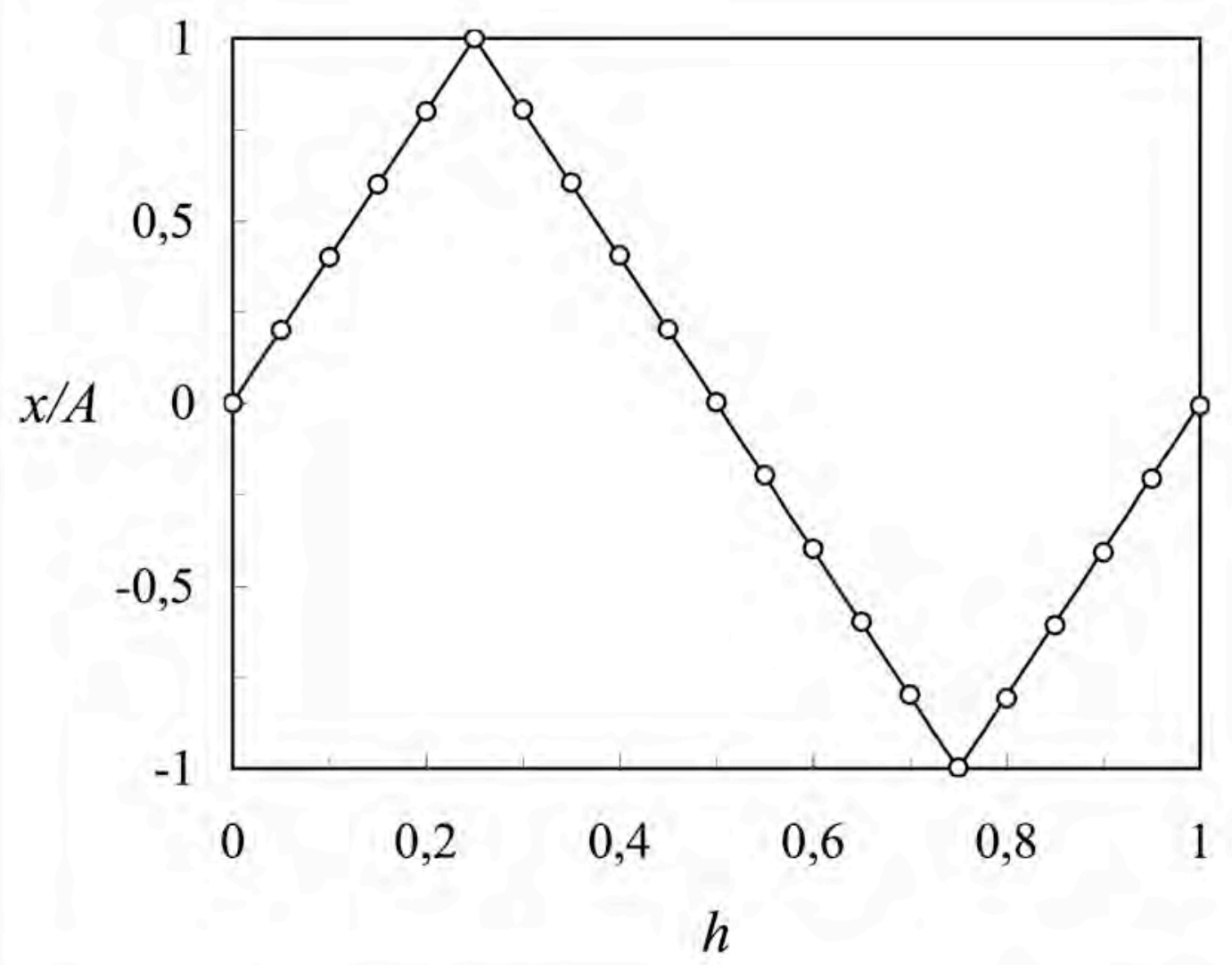


FIGURE 8

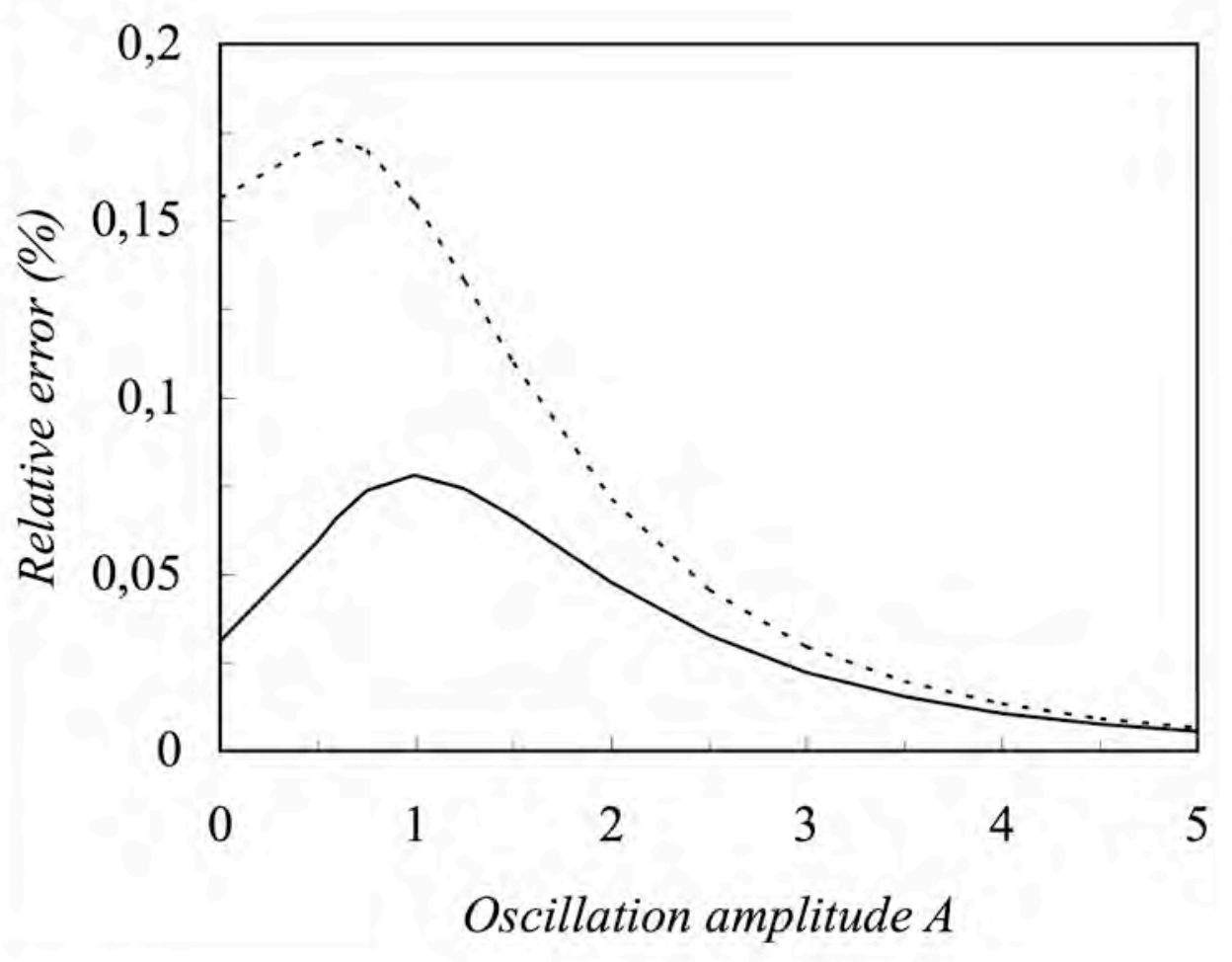


FIGURE 9

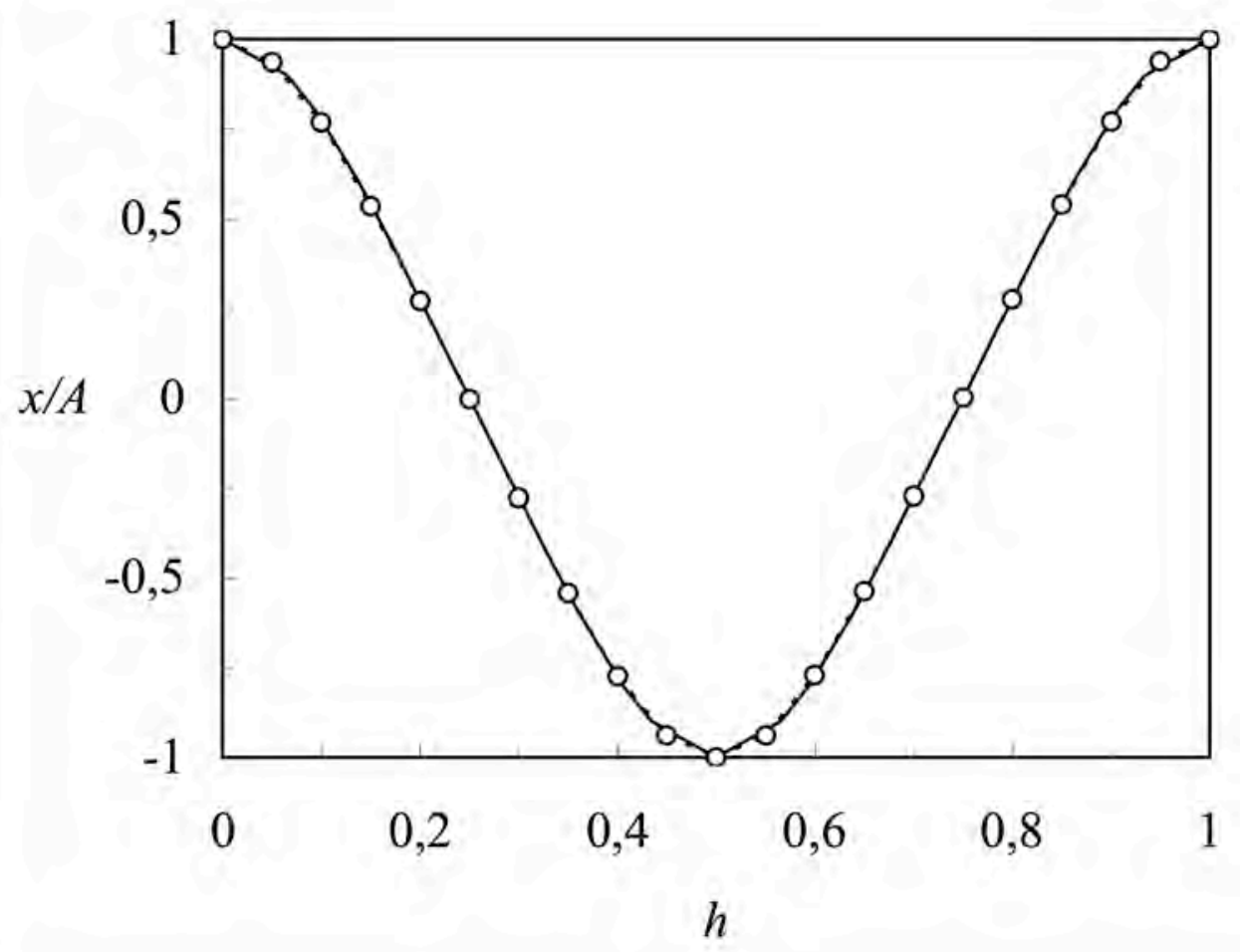

